TRANSACTIONS OF THE

AMERICAN MATHEMATICAL SOCIETY

Volume 352, Number 2, Pages 525-552

S 0002-9947(99)02260-6

Article electronically published on September 10, 1999

\title{
QUANTIZATION OF PRESYMPLECTIC MANIFOLDS AND CIRCLE ACTIONS
}

\author{
ANA CANNAS DA SILVA, YAEL KARSHON, AND SUSAN TOLMAN
}

\begin{abstract}
We prove several versions of "quantization commutes with reduction" for circle actions on manifolds that are not symplectic. Instead, these manifolds possess a weaker structure, such as a $\operatorname{spin}^{c}$ structure. Our theorems work whenever the quantization data and the reduction data are compatible; this condition always holds if we start from a presymplectic (in particular, symplectic) manifold.
\end{abstract}

\section{INTRODUCTION}

Morally, quantization is a functor that associates a Hilbert space to every symplectic manifold and linear operators to functions, subject to certain conditions suggested by physics. In particular, quantization should associate to every Hamiltonian action of a group on a symplectic manifold, a unitary representation of that group. Symplectic reduction models the fact that symmetries of a Hamiltonian dynamical system enable one to reduce the number of degrees of freedom of the system; it produces a lower dimensional symplectic manifold. In the last few years there has been much progress around a "conjecture" of Guillemin and Sternberg [GS] that can be loosely phrased as quantization commutes with reduction. This means that the quantization of the reduced space ought to be naturally isomorphic to the space of fixed vectors in the quantization of the original manifold. In this paper we show that the assertion "quantization commutes with reduction" makes sense and is true in a much more general context than that of symplectic manifolds. To make this statement precise, both quantization and reduction must be rigorously defined. To make this statement true, some compatibility relations between the data for quantization and the data for reduction must be assumed.

Although it is impossible to satisfy all the requirements of quantization, some recipes are partially successful. For example, let a group $G$ act in a Hamiltonian fashion on a Kähler manifold $(M, \omega)$. Suppose that $\omega$ is integral, and let $L$ be a $G$-equivariant holomorphic line bundle with first Chern class $[\omega]$. The space of global holomorphic sections of $L$ is a representation of $G$; this is called the Kähler quantization.

Received by the editors September 26, 1997.

1991 Mathematics Subject Classification. Primary 58G10, 81S10; Secondary 58F06, 53C15.

A. Cannas da Silva was partially supported by a NATO fellowship. Her research at MSRI was supported in part by NSF grant DMS 9022140. Y. Karshon was partially supported by NSF grant DMS 9404404. S. Tolman was partially supported by an NSF postdoctoral fellowship. 
The first natural extension of this idea, suggested by Bott [B1], is to replace the space of holomorphic sections by the alternating sum of sheaf cohomology groups, $\sum_{i}(-1)^{i} H^{i}\left(M, \mathcal{O}_{L}\right)$, where $\mathcal{O}_{L}$ is the sheaf of germs of holomorphic sections of $L$. By Kodaira's theorem, $H^{i}\left(M, \mathcal{O}_{L^{n}}\right)=0$ for all $i \geq 1$ for sufficiently large $n$, and we retrieve Kähler quantization in the semi-classical limit. This alternating sum is a virtual representation of $G$, i.e., a formal difference of two linear representations. By Dolbeault's theorem, it is the same as the index of the Dolbeault $\bar{\partial}$ operator with coefficients in $L$.

A second generalization, that we will call almost-Kähler quantization ${ }^{1}$, applies to symplectic manifolds $(M, \omega)$ on which a group $G$ acts in a Hamiltonian fashion. This uses the existence and uniqueness, up to homotopy, of an almost complex structure $J$ that is compatible with $\omega$, i.e., such that the bilinear form

$$
g_{p}(v, w)=\omega_{p}\left(J_{p} v, w\right) ; \quad v, w \in T_{p} M
$$

is symmetric and positive definite for all $p \in M$. (When $J$ is actually a complex structure, compatibility is equivalant to $\omega$ being a Kähler form.) If $\omega$ is integral, there is a complex line bundle $L$ with first Chern class [ $\omega]$. Choosing additional auxiliary data, this determines an elliptic operator, $\mathcal{D}$, whose index only depends on $\omega$ and $J$. (Bott calls it the "rolled-up Dolbeault operator", whereas Duistermaat coined the term "Dolbeault-Dirac operator".) Almost-Kähler quantization is defined to be the index of this operator:

$$
[\operatorname{ker} \mathcal{D}]-[\operatorname{coker} \mathcal{D}]
$$

The kernel and cokernel of $\mathcal{D}$ are linear $G$-representations. ${ }^{2}$ The index, as a virtual representation, is determined by its character function, $\chi: G \rightarrow \mathbb{C}$, given by $\chi(g)=$ $\operatorname{Trace}\left(\left.g\right|_{\text {ker } \mathcal{D}}\right)-\operatorname{Trace}\left(\left.g\right|_{\text {coker } \mathcal{D}}\right)$. The multiplicity of $a$ in the index is its multiplicity in $\operatorname{ker} \mathcal{D}$ minus its multiplicity in coker $\mathcal{D}$. Guillemin and Sternberg observed that the Riemann-Roch number, which computes this index, is in fact a symplectic invariant, and hence can be used to quantize a symplectic manifold; see Vergne [V1] and Guillemin $[\mathrm{G}]$.

One can also define quantization as the index of other differential operators, such as the Dirac spin or $\operatorname{spin}^{c}$ operators. Bott used the Dirac $\operatorname{spin}^{c}$ operator in this context back in the 60's [B2]. Representation theorists, attempting to construct irreducible representations by quantizing coadjoint orbits, used the Dirac spin operator in the 1970's; see, e.g., [P] and references therein. Duflo preferred the spin ${ }^{c}$ operator for this purpose.

We begin our paper by discussing, in section 2, different possible extensions of symplectic quantization to non-symplectic settings. We work with each of the following quantization data (in increasing generality):

1. an almost complex structure and a complex line bundle;

2. a stable complex structure, an orientation, and a complex line bundle; or

3. a $\operatorname{spin}^{c}$ structure.

Each such data determines a Dirac operator, $\mathcal{D}$, which acts on the sections of certain vector bundles over $M$. The corresponding quantization is defined to be the index

\footnotetext{
${ }^{1}$ Many authors call this $\operatorname{spin}^{c}$ quantization, but we prefer to reserve that name for the quantization defined on all $\operatorname{spin}^{c}$ structure.

${ }^{2}$ Recently, Borthwick and Uribe [BU] have proved vanishing theorems for the cokernels, showing that almost-Kähler quantization yields honest vector spaces semiclassically.
} 
of this operator. The definition of the Dirac operator requires some additional structure (connection, metric), but its index is independent of these choices.

Let a compact Lie group $G$ act on a symplectic manifold $(M, \omega)$ with a moment map $\Phi: M \rightarrow \mathfrak{g}^{*}$, where $\mathfrak{g}^{*}$ is the dual to the Lie algebra of $G$. For every regular value $a \in \mathfrak{g}^{*}$, the reduced space, $M_{\text {red }}^{a}:=\Phi^{-1}(G \cdot a) / G$, is naturally a symplectic orbifold, and it is a symplectic manifold when $G$ acts freely [MW]. In section 3 we show how $S^{1}$-equivariant quantization data descends to quantization data on the reduced space. In the absence of a moment map and its level sets, we define the reduced space to be the quotient by the circle action of an arbitrary invariant hypersurface that splits our manifold into two pieces.

Guillemin and Sternberg proved that Kähler quantization commutes with reduction in the same paper where they stated their conjecture [GS]. More specifically, they proved that the dimension of the quantization of the reduced space at $a=0$ is equal to the multiplicity of 0 in the quantization of the original manifold. Recently, much progress has been made in proving that almost-Kähler quantization commutes with symplectic reduction under various assumptions; Duistermaat, Guillemin, Jeffrey, Kirwan, Meinrenken, Sjamaar, Vergne and Wu ([M1], [DGMW], $[\mathrm{G}],[\mathrm{JK}],[\mathrm{V} 2],[\mathrm{M} 2],[\mathrm{MS}]$ ) covered the cases where one starts with a symplectic orbifold, where the level for reduction is singular, and where the group acting is an arbitrary compact Lie group. A survey of these developments, as well as an excellent introduction to the problem, can be found in [S].

We extend this theorem by proving that our three versions of quantization commute with our generalized notion of reduction as long as certain components of the fixed point set lie on the correct side of the splitting hypersurface. The precise condition depends on the quantization data; see section 4 . Our main theorem, that $\operatorname{spin}^{c}$ quantization commutes with reduction, implies the corresponding theorems for stable complex and almost complex quantization. Hence it implies the standard theorem for almost-Kähler quantization. ${ }^{3}$

Each of these three types of quantization data gives a presymplectic form (that is, a closed two-form $)^{4}$ on our manifold; more precisely, each gives the cohomology class represented by such a form. We think of the quantization as a quantization of the presymplectic manifold and of the quantization data as a "polarization" auxiliary data used to define the quantization. Although the presymplectic forms are not essential for quantization neither reduction nor "quantization commutes with reduction"; they are relevant because traditionally quantization and reduction only applied to symplectic manifolds, and because presymplectic forms provide interesting examples.

Presymplectic forms come up naturally in many mathematical contexts: as curvature forms of line bundles, as pull-backs of Kähler forms via complex blow-ups, on connected sums of symplectic manifolds, as the averages of symplectic forms by group actions [GK1], as folding type structures [CGW], and in the framework of symplectic cobordism [GGK]. For an application to representation theory see [GK1]. Presymplectic forms also come up in physics [GNH].

To a group action on a presymplectic manifold one can associate a moment map, just as one does on a symplectic manifold. The zero level set of the moment map

\footnotetext{
${ }^{3}$ Note that other authors use the term "spin ${ }^{c}$ quantization" to refer only to this very special case.

${ }^{4}$ We do not insist, as some authors do, that the closed two-form have constant rank.
} 
is a hypersurface that automatically satisfies the compatibility conditions mentioned earlier. Thus "presymplectic quantization commutes with reduction". We provide the details in section 5 . This theorem was proven in the special case of almost-Kähler quantization of toric manifolds in $[\mathrm{KT}]$, then generalized to completely integrable torus actions and $\operatorname{spin}^{c}$ quantization in [GK2].

In sections 6 and 7 we prove our main theorem, that $\operatorname{spin}^{c}$ quantization commutes with reduction. Our proof is modeled on the simple and elegant proof of Duistermaat-Guillemin-Meinrenken-Wu [DGMW] for the symplectic case. The result follows from applying the index theorem of Atiyah-Segal-Singer to the original space, to the "Lerman cut space" (see section 6), and to the reduced space, and comparing terms.

$\operatorname{Spin}^{c}$ quantization is our favorite, for several reasons: $\operatorname{spin}^{c}$ quantization of a presymplectic manifold depends on no auxiliary data; it only depends on the manifold, the orientation, and the cohomology class of the two-form. The theorem that "quantization commutes with reduction" is the strongest in the $\operatorname{spin}^{c}$ case; as mentioned earlier, it implies similar theorems for almost complex and stable complex quantization. For presymplectic manifolds, the $\operatorname{spin}^{c}$ theorem is the most robust in that it gives the biggest freedom to choose the level set at which we can reduce; see Remarks 4.11 and 5.5.

We have designed this paper so that the reader who is only interested in the $\operatorname{spin}^{c}$ case does not have to read about the other cases. To read the paper this way, simply skip all the subsections with the word "complex" in the title, also skip Theorems 1 and 2, the remarks immediately after them, and their proofs in section 4 , and additionally skip Corollaries 1 and 2 in section 5 .

In this paper we restrict to circle actions on manifolds and insist that the circle act freely on the reducible hypersurface, so that the reduced space is a smooth manifold. However, we expect our results to generalize to torus actions and to the case where the original space is an orbifold or the action is only locally free.

As for actions of nonabelian groups, the most straightforward generalization of the theorem is true in the symplectic case [M2] but is false in the almost complex, stable complex, and $\operatorname{spin}^{c}$ presymplectic cases; counterexamples have been found by Vergne and by Vogan for the action of $S U(2)$ on $\mathbb{C P}^{1}$. (See $[\mathrm{MS}]$.) The general presymplectic nonabelian case was recently resolved by Tolman $[\mathrm{T}]$.

\section{ACKNOWLEDGEMENTS}

The authors wish to acknowledge their gratitude for the useful discussions, suggestions, and support of Victor Guillemin, Viktor L. Ginzburg, and Eckhard Meinrenken.

\section{Quantization}

In this section we describe several ways to extend almost-Kähler quantization (see section 1) and to quantize non-symplectic manifolds via indices of elliptic differential operators. This is mostly review of known material, but is necessary for the rest of the paper. In $\S 2.4$ we interpret these as quantizations of presymplectic manifolds.

2.1. Almost complex quantization. Let $M$ be a compact manifold, $L \rightarrow M$ a complex line bundle, and $J: T M \rightarrow T M$ an almost complex structure. Choose a metric on $M$, a Hermitian structure on $L$, and a Hermitian connection on $L$. This data determines an elliptic operator, $\mathcal{D}$, called the twisted Dolbeault-Dirac 
operator (see $[\mathrm{GK} 2, \S 9]$ or $[\mathrm{D}])$. The almost complex quantization of $(M, L, J)$ is the index of this operator:

$$
Q(M, L, J):=[\operatorname{ker} \mathcal{D}]-[\operatorname{coker} \mathcal{D}] .
$$

If the above data is equivariant with respect to an action of a Lie group $G$, the index is a virtual representation of $G$. The index is independent of the auxiliary choices of metric and connection.

2.2. Spin $^{c}$ quantization. The group $\operatorname{spin}(n)$ is the connected double cover of the group $\mathrm{SO}(n)$. Let $q: \operatorname{spin}(n) \rightarrow \mathrm{SO}(n)$ be the covering map, and let $\{1, \epsilon\}$ be its kernel. The group $\operatorname{spin}^{c}(n)$ is the quotient $(\operatorname{spin}(n) \times U(1)) / \mathbb{Z}_{2}$, where $\mathbb{Z}_{2}$ is generated by $(\epsilon,-1)$. There are two canonical group homomorphisms:

$$
\begin{aligned}
& f: \operatorname{spin}^{c}(n) \rightarrow \mathrm{SO}(n) \quad \text { and } \quad \operatorname{det}: \operatorname{spin}^{c}(n) \rightarrow U(1) \\
& {[s, u] \mapsto q(s) \quad[s, u] \mapsto u^{2}}
\end{aligned}
$$

(the square brackets indicate class modulo $\mathbb{Z}_{2}$ ).

Let $V \rightarrow M$ be a real oriented vector bundle of rank $n$, equipped with a fiber metric, ${ }^{5}$ and let $\mathcal{F}(V)$ be its oriented orthonormal frame bundle. The group $\operatorname{spin}^{c}(n)$ acts on $\mathcal{F}(V)$ from the right via the homomorphism $f$. A spin ${ }^{c}$ structure on the vector bundle $V$ is a $\operatorname{spin}^{c}(n)$-principal bundle, $P \rightarrow M$, together with a $\operatorname{spin}^{c}(n)$-equivariant bundle map, $p: P \rightarrow \mathcal{F}(V)$. The line bundle $L=P \times_{\operatorname{det}} \mathbb{C}$ is called the line bundle associated to $\boldsymbol{P}$. A $\operatorname{spin}^{c}$ structure on an oriented Riemannian manifold is a $\operatorname{spin}^{c}$ structure on its tangent bundle.

If a group $G$ acts on the bundle $V$, preserving the fiber metric, we define an equivariant $\operatorname{spin}^{c}$ structure on $V$ by requiring $P$ to be a $G$-equivariant principal bundle and the map $p$ to be $\left(G \times \operatorname{spin}^{c}(n)\right)$-equivariant.

A $\operatorname{spin}^{c}$ structure $(P, p)$ on a compact oriented Riemannian manifold $M$, plus a connection on $P$, determines an elliptic operator, $\mathcal{D}$, called the $\operatorname{spin}^{c}$-Dirac operator (see $[\mathrm{GK} 2, \S 9]$ or $[\mathrm{D}])$. The $\operatorname{spin}^{c}$ quantization of $(M, P, p)$ is the index of this operator:

$$
Q(M, P, p):=[\operatorname{ker} \mathcal{D}]-[\operatorname{coker} \mathcal{D}] .
$$

If $(P, p)$ is a $G$-equivariant $\operatorname{spin}^{c}$ structure, and the connection on $P$ is $G$-invariant, the index is a virtual $G$-representation. The index is independent of the choice of connection on $P$.

Lemma 2.2. Let $M$ be an oriented Riemannian manifold with a $G$ action. There is an equivariant $\operatorname{spin}^{c}$ structure on $M$ naturally associated to every invariant almost complex structure on $M$ and equivariant line bundle $L$ over $M$. The line bundle associated to this $\operatorname{spin}^{c}$ structure is $L \otimes L \otimes K^{*}$, where $K$ is the canonical line bundle, $\bigwedge_{\mathbb{C}}^{n} T^{*} M$. The $\operatorname{spin}^{c}$ quantization then coincides with the almost complex quantization.

Proof. Apply the standard construction (see [LM], appendix D, example D.6) to the tangent bundle $T M$. The quantizations are the same because the DolbeaultDirac operator and the $\operatorname{spin}^{c}$-Dirac operator $\mathcal{D}$ have the same principal symbol and hence the same index; see $[\mathrm{D}, \S 5]$.

\footnotetext{
${ }^{5}$ It would be more elegant, but less standard, to follow Duflo and Vergne in using quantum line bundles (see [V2]) instead of $\operatorname{spin}^{c}$ structures. These eliminate the unnecessary choice of a metric.
} 
Remark 2.3. There are two different elliptic operators on an even dimensional $\operatorname{spin}^{c}$ manifold: $D^{+}: S^{+} \rightarrow S^{-}$and $D^{-}=\left(D^{+}\right)^{*}: S^{-} \rightarrow S^{+}$, where $S^{+}$ and $S^{-}$are the so-called positive and negative spinors. In dimensions $2 \bmod 4$, there is no universally recognized standard as to which half of the spinor bundle should be called $S^{+}$. We follow the conventions of Lawson-Michelson [LM], Duistermaat $[D]$, and the majority of recent authors. Unfortunately, these disagree with Atiyah-Singer [ASIII] and Vergne [V2], and thus our formulas will differ from those in [ASIII] by $(-1)^{n}$ in dimension $2 n$.

We will use the following lemma twice: to define the quantization of a stable complex structure in $\S 2.3$, and to define the reduction of a $\operatorname{spin}^{c}$ structure in $\S 3$.

Lemma 2.4. Let $V \rightarrow M$ be a vector bundle equipped with a fiber metric and orientation and with a left action of a Lie group $G$. Every equivariant spin ${ }^{c}$ structure on a direct sum of $V$ with an equivariantly trivial bundle naturally induces an equivariant spin ${ }^{c}$ structure on $V$, with the same associated line bundle.

Proof. Let $n=\operatorname{rank}(V)$. The splitting $\mathbb{R}^{n+k}=\mathbb{R}^{n} \times \mathbb{R}^{k}$ induces the following commuting diagrams of group homomorphisms:

$$
\begin{array}{cccc}
\operatorname{spin}^{c}(n) & \hookrightarrow & \operatorname{spin}^{c}(n+k) \\
f \downarrow & & f \downarrow \\
\mathrm{SO}(n) & \hookrightarrow & \mathrm{SO}(n+k)
\end{array}
$$

and

$$
\begin{array}{ccc}
\operatorname{spin}^{c}(n) & \hookrightarrow & \operatorname{spin}^{c}(n+k) \\
\operatorname{det} \downarrow & & \operatorname{det} \downarrow \\
U(1) & \rightrightarrows & U(1)
\end{array}
$$

Let $\left(P^{\prime}, p^{\prime}\right)$ be an equivariant $\operatorname{spin}^{c}$ structure on $V \oplus \mathbb{R}^{k}$. The bundle $P$, which is defined by the following pull-back diagram,

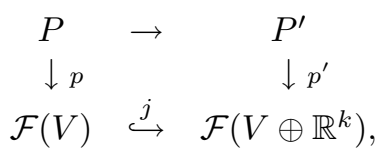

is a $G$-equivariant $\operatorname{spin}^{c}(n)$-principal bundle over $M$. The map $p:=j^{*} p^{\prime}$ is $\left(G \times \operatorname{spin}^{c}(n)\right)$-equivariant by $(2.5)$. By (2.6), the line bundle associated to $P$ is the same as that associated to $P^{\prime}$.

2.3. Stable complex quantization. Stable complex structures are a generalization of almost complex structures which behave much better under reduction; see $\S 3$.

A stable complex structure on a manifold $M$ is an equivalence class of complex structures on the vector bundles $T M \oplus \mathbb{R}^{k}$; two such complex structures, on bundles $E_{1}=T M \oplus \mathbb{R}^{k_{1}}$ and $E_{2}=T M \oplus \mathbb{R}^{k_{2}}$, are equivalent if there exist $r_{1}, r_{2}$ such that $E_{1} \oplus \mathbb{C}^{r_{1}}$ and $E_{2} \oplus \mathbb{C}^{r_{2}}$ are isomorphic complex vector bundles. When a group acts on $M$ we define an equivariant stable complex structure by requiring the complex structures to be invariant and the isomorphism to be equivariant. Here the group acts on $T M$ by the natural lifting of its action on $M$, and it acts trivially on the trivial bundles $\mathbb{R}^{k}$ and $\mathbb{C}^{r}$. The canonical line bundle of a stable complex structure is the top wedge $K=\bigwedge_{\mathbb{C}}^{m} E^{*}$, where $E=T M \oplus \mathbb{R}^{k}$ is a complex vector bundle of rank $m$ representing the stable complex structure. 
An almost complex structure determines a stable complex structure and an orientation. A stable complex structure does not determine an orientation. ${ }^{6}$

Lemma 2.7. Let $M$ be an oriented Riemannian manifold with a $G$ action. There is an equivariant $\operatorname{spin}^{c}$ structure on $M$ naturally associated to every invariant stable complex structure on $M$ and equivariant line bundle $L$ over $M$. The line bundle associated to this $\operatorname{spin}^{c}$ structure is $L \otimes L \otimes K^{*}$, where $K$ is the canonical line bundle of the stable complex structure.

Proof. The standard construction (see [LM], appendix D, example D.6), applied to a complex vector bundle that represents the stable complex structure, yields a $\operatorname{spin}^{c}$ structure on the vector bundle $T M \oplus \mathbb{R}^{k}$ with associated line bundle $L \otimes L \otimes K^{*}$. By Lemma 2.4, this $\operatorname{spin}^{c}$ structure determines a $\operatorname{spin}^{c}$ structure on $T M$ with the same associated line bundle. Different representatives of the stable complex class yield isomorphic $\operatorname{spin}^{c}$ structures by naturality of the standard construction in [LM] and of the pull-back diagram in the proof of Lemma 2.4.

Remark 2.8. In passing from an almost complex or stable complex structure and a line bundle to the corresponding $\operatorname{spin}^{c}$ structure we lose information. For example, on $\mathbb{C P}^{1} \times \mathbb{C P}^{1}$ the line bundle $O(m) \otimes O(m)$ with the standard complex structure, and the line bundle $O(m+2) \otimes O(m+2)$ with the opposite complex structure induce the same $\operatorname{spin}^{c}$ structure.

Let $M$ be a compact manifold with an action of a compact Lie group $G, L$ an equivariant complex line bundle over $M, \mathcal{J}$ an equivariant stable complex structure on $M$, and $\mathcal{O}$ an orientation on $M$. Let $(P, p)$ be the corresponding $\operatorname{spin}^{c}$ structure described in Lemma 2.7 (for an arbitrary Riemannian metric on $M$ ), and let $\mathcal{D}$ be the corresponding $\operatorname{spin}^{c}$-Dirac operator (for an arbitrary connection on $P$ ). Stable complex quantization is the index of this operator, i.e., is the virtual $G$-representation

$$
Q(M, L, \mathcal{J}, \mathcal{O})=[\operatorname{ker} \mathcal{D}]-[\operatorname{coker} \mathcal{D}]
$$

Remark 2.9. Let $J$ be an almost complex structure on $M$ and $L$ a complex line bundle over $M$. Let $\mathcal{J}$ and $\mathcal{O}$ be the stable complex structure and the orientation induced by $J$. The $\operatorname{spin}^{c}$ structure associated to the data $(M, L, J)$ by Lemma 2.2 coincides with the $\operatorname{spin}^{c}$ structure associated to the data $(M, L, \mathcal{J}, \mathcal{O})$ by Lemma 2.7. Consequently, the almost complex quantization of $(M, L, J)$ is the same as the stable complex quantization of $(M, L, \mathcal{J}, \mathcal{O})$.

2.4. Quantization of presymplectic manifolds. A presymplectic manifold is a manifold $M$ equipped with a presymplectic form $\omega$, i.e., a closed, possibly degenerate, two-form. Let the circle group, $S^{1}$, act on $M$ and preserve $\omega$. A moment map is a map $\Phi: M \rightarrow \mathbb{R}$ such that $d \Phi=\iota\left(\eta_{M}\right) \omega$, where $\eta$ the positive primitive lattice element in $\operatorname{Lie}\left(S^{1}\right)$, and $\eta_{M}$ is the vector field on $M$ which generates the action of $\exp (t \eta)$, for $t \in \mathbb{R}$. (See Appendix A for an explanation of our conventions.)

Given a moment map $\Phi$, we can define the quantization of $(M, \omega, \Phi)$ using each of the three types of quantization above. For this we need to impose an integralitytype condition on $\omega$ and $\Phi$; the precise condition depends on whether we perform (almost or stable) complex quantization or $\operatorname{spin}^{c}$ quantization.

\footnotetext{
${ }^{6}$ For instance, the almost complex structures $J$ and $-J$ on $\mathbb{C}$ define the same stable complex structure but opposite orientations.
} 
Almost complex and stable complex quantizations. The triple $(M, \omega, \Phi)$ is complex prequantizable if the equivariant cohomology $\operatorname{class}^{7}[\omega+u \Phi]$ is integral. This condition precisely guarantees that we can find an equivariant line bundle $L$ whose equivariant first Chern class is $[\omega+u \Phi]$. (The non-equivariant case is explained well in [Ko]. By applying that to $M \times_{S^{1}} E S^{1}$, we get the equivariant case.)

Given an invariant almost complex structure $J$ on $M$, the almost complex quantization of the presymplectic manifold is

$$
Q(M, \omega, \Phi, J):=Q(M, L, J) .
$$

Given an equivariant stable complex structure $\mathcal{J}$ on $M$ and an orientation $\mathcal{O}$ on $M$, the stable complex quantization of the presymplectic manifold is

$$
Q(M, \omega, \Phi, \mathcal{J}, \mathcal{O}):=Q(M, L, \mathcal{J}, \mathcal{O})
$$

Although there may exist different line bundles with the same equivariant first Chern class, the quantizations (2.10) and (2.11) are independent of this choice. This follows from the Atiyah-Segal-Singer formula for the equivariant index; see $\S 7$.

$\operatorname{Spin}^{c}$ quantization. The triple $(M, \omega, \Phi)$ is spin $^{\mathbf{c}}$ prequantizable if the equivariant cohomology class $2[\omega+u \Phi]$ is integral and the modulo 2 reduction of $2[\omega+u \Phi]$ is the second equivariant Stiefel-Whitney class of $M$. For any orientation $\mathcal{O}$ and Riemannian metric on $M$, this condition precisely guarantees that there exists a $G$-equivariant $\operatorname{spin}^{c}$ structure $(P, p)$ on $M$ such that the equivariant first Chern class of its associated line bundle is $2[\omega+u \Phi]$; see [LM]. The $\operatorname{spin}^{c}$ quantization of the presymplectic manifold is defined to be

$$
Q(M, \omega, \Phi, \mathcal{O}):=Q(M, P, p) .
$$

It is independent of the the metric on $M$ and the $\operatorname{spin}^{c}$ structure $(P, p)$. This follows from the Atiyah-Segal-Singer formula for the equivariant index; see $\S 7$.

Remark 2.13. Let $S^{1}$ act on $M$, and consider a $\operatorname{spin}^{c}$ prequantizable equivariant two-form, $\omega+u \Phi$. For any weight $a \in \mathbb{Z}$, the equivariant two-form $\omega+u(\Phi-a)$ is also spin $^{c}$ prequantizable; its quantization differs from that of $(M, \omega, \Phi, \mathcal{O})$ by tensoring with the one-dimensional $S^{1}$-representation with weight $a$. Hence the multiplicity of $a$ in $Q(M, \omega, \Phi, \mathcal{O})$ is equal to the multiplicity of 0 in $Q(M, \omega, \Phi-a, \mathcal{O})$. This fact is needed to make the shifting trick work, and hence to make quantization commute with reduction at all weights; see section 5 . Note that this remark would be false without the factor of 2 in the definition of $\operatorname{spin}^{c}$ quantization.

An analogous remark holds for almost and stable complex structures, that is, the multiplicity of $a$ in $Q(M, \omega, \Phi, J)$ is the multiplicity of 0 in $Q(M, \omega, \Phi-a, J)$, etc.

Remark 2.14. A line bundle $L$ and an almost (or stable) complex structure $J$ on $M$ naturally determine a $\operatorname{spin}^{c}$ structure $(P, p)$ such that the quantizations $Q(M, L, J)$ and $Q(M, P, p)$ coincide. Nevertheless, we interpret these as the quantizations of two different presymplectic forms. Specifically, $Q(M, L, J)$ is the quantization of a two-form representing the first Chern class of $L$, whereas $Q(M, P, p)$ is the quantization of a two-form representing the first Chern class of $L$ plus half the first Chern class of the dual to the canonical bundle of the almost complex structure. (See Lemma 2.2 and Example 2.15.)

\footnotetext{
${ }^{7}$ Here, $u$ is a formal variable. For definitions of equivariant forms and equivariant cohomology, please see $[\mathrm{BGV}]$.
} 
Example 2.15. Let $[\sigma] \in H^{2}\left(\mathbb{C P}^{2}\right)$ generate the integral cohomology. The dual to the canonical bundle has first Chern class $3[\sigma]$. A presymplectic form $\omega$ is $\operatorname{spin}^{c}-$ quantizable if and only if $[\omega]=(k+3 / 2)[\sigma]$ for some $k \in \mathbb{Z}$; in particular, the $\operatorname{spin}^{c}$-quantizable two-forms are not integral. The $\operatorname{spin}^{c}$ quantization of $(k+3 / 2) \sigma$ coincides with the almost complex quantization of $k \sigma$.

\section{REDUCTION}

Let the circle act on a manifold $M$. When $M$ possesses a symplectic form that is invariant under the action and has a moment map $\Phi: M \rightarrow \mathbb{R}$, symplectic reduction produces a symplectic structure on the reduced space $M_{\mathrm{red}}^{a}=\Phi^{-1}(a) / S^{1}$ for every regular value $a$ (see $[\mathrm{MW}]$ ). We generalize this idea in two ways: first, we take the quotient of arbitrary co-oriented hypersurfaces on which the circle acts freely. Second, we describe how additional equivariant structures on $M$ descend to this subquotient of $M$.

Definition 3.1. A reducible hypersurface in $M$ is a co-oriented submanifold $Z$ of codimension one that is invariant under the $S^{1}$ action and on which this action is free. The reduction of $\boldsymbol{M}$ at $\boldsymbol{Z}$ is the quotient of $Z$ by the circle action, $M_{\text {red }}:=Z / S^{1}$.

It is useful to keep in mind the diagram

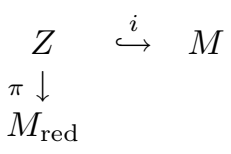

where $i$ and $\pi$ are the inclusion and quotient maps, respectively.

Definition 3.3. A reducible hypersurface $Z$ is splitting if its complement, $M \backslash Z$, is a disjoint union of two (not necessarily connected) open pieces, $M_{+}$and $M_{-}$, such that positive normal vectors to $Z$ point into $M_{+}$and negative normal vectors point into $M_{-}$. We then say that $Z$ splits $M$ into $M_{+}$and $M_{-}$.

Example 3.4. Let $\Phi: M \rightarrow \mathbb{R}$ be a smooth $S^{1}$-invariant function. Assume that 0 is a regular value for $\Phi$ and that $S^{1}$ acts freely on the level set $\Phi^{-1}(0)$. Then $Z=\Phi^{-1}(0)$ is a reducible hypersurface, and it splits $M$ into $M_{+}:=\Phi^{-1}(0, \infty)$ and $M_{-}:=\Phi^{-1}(-\infty, 0)$. Conversely, every reducible splitting hypersurface can be obtained in this way.

3.1. Reduction and tangent bundles. Let $i: Z \rightarrow M$ be the inclusion of a reducible hypersurface. We have the following short exact sequences of vector bundles over $Z$ :

$$
0 \rightarrow T Z \rightarrow i^{*}(T M) \rightarrow N Z \rightarrow 0
$$

and

$$
0 \rightarrow T \mathcal{S} \rightarrow T Z \rightarrow \pi^{*}\left(T M_{\text {red }}\right) \rightarrow 0
$$

where $i$ and $\pi$ are the inclusion and quotient maps, as in (3.2). Here $T \mathcal{S}$ is the sub-bundle of $T Z$ consisting of those vectors that are tangent to the $S^{1}$ orbits; it is an oriented trivial real line bundle. Similarly, $N Z$, the normal bundle to $Z$ in $M$, is an oriented trivial real line bundle, because $Z$ is co-oriented. 
It follows from (3.5) and (3.6) that there exists a (non-canonical) isomorphism of vector bundles over $Z$ :

$$
i^{*}(T M) \cong \pi^{*}\left(T M_{\text {red }}\right) \oplus T \mathcal{S} \oplus N Z .
$$

This isomorphism is $S^{1}$-equivariant with respect to the natural $S^{1}$ action on the bundle $\pi^{*}\left(T M_{\text {red }}\right)$ and the trivial $S^{1}$ action on $T \mathcal{S} \oplus N Z$. If we choose trivializations of $N Z$ and $T \mathcal{S},(3.7)$ gives an equivariant isomorphism:

$$
i^{*}(T M) \cong \pi^{*}\left(T M_{\text {red }}\right) \oplus \mathbb{R}^{2}
$$

where $\mathbb{R}^{2}$ denotes the trivial bundle over $Z$ with fiber $\mathbb{R}^{2}$.

3.2. Reduction of orientations. An orientation $\mathcal{O}$ on $M$ determines a reduced orientation $\mathcal{O}_{\text {red }}$ on $M_{\text {red }}$ by requiring the induced orientations on the left and right sides of (3.7) to agree.

This convention guarantees that if $\mathcal{O}$ is the orientation induced by a symplectic form on $M$ and $Z$ is the zero level set of a moment map, then $\mathcal{O}_{\text {red }}$ is the orientation induced by the reduced symplectic form (see $\S 3.7$ ).

3.3. Reduction of line bundles. An equivariant complex line bundle $L$ over $M$ determines a reduced line bundle $L_{\text {red }}:=i^{*} L / S^{1}$ over $M_{\text {red }}:=Z / S^{1}$, where $i: Z \rightarrow M$ is the inclusion of a reducible hypersurface.

3.4. Reduction of almost complex structures. Almost complex structures do not always descend to the reduced space, but they do when the following condition is satisfied.

Definition 3.9. A reducible hypersurface $Z$ is admissible for an invariant almost complex structure $J$ on $M$ if $J \xi_{M}$ is transverse to $Z$ at all points of $Z$, where $\xi_{M}$ is the vector field on $M$ that generates the circle action.

Given an admissible hypersurface $Z$ for an invariant almost complex structure $J$, one can construct an almost complex structure on the reduced space $M_{\text {red }}=Z / S^{1}$ as follows. Let $\mathcal{V}:=T Z \cap J(T Z) \subset i^{*}(T M)$ be the maximal complex sub-bundle of $T Z$. The composition $\mathcal{V} \hookrightarrow T Z \stackrel{\pi_{*}}{\rightarrow} \pi^{*}\left(T M_{\text {red }}\right)$ is an isomorphism. This isomorphism defines an equivariant complex structure on the vector bundle $\pi^{*}\left(T M_{\text {red }}\right)$, which in turn descends to an almost complex structure $J_{\text {red }}$ on $M_{\text {red }}$.

If $Z$ is an admissible hypersurface, we assign a sign to each connected component, $X$, of the reduced space, $M_{\text {red }}:=Z / S^{1}$ :

$$
\sigma_{J}(X)=\left\{\begin{aligned}
1 & \text { if } J \xi_{M} \text { points in the positive direction at points of } \pi^{-1}(X), \\
-1 & \text { if } J \xi_{M} \text { points in the negative direction at points of } \pi^{-1}(X),
\end{aligned}\right.
$$

where "positive" and "negative" are with respect to the given co-orientation of $Z$.

Example 3.11. Suppose $(M, \omega)$ is a symplectic manifold and $J$ is a compatible almost complex structure. If a level set of the moment map for a Hamiltonian circle action is reducible, then it is admissible, and $\sigma_{J}\left(M_{\text {red }}\right)=+1$.

More generally, let $\omega$ be presymplectic and let $(M, J, \omega)$ have the property that for every $v \in T M, \omega(v, J v)=0$ implies $\iota(v) \omega=0$; equivalently, "if $v$ has any friends, $J v$ is a friend". This property holds if, for example, $(M, J)$ is the complex 
blowup of a Kähler manifold along some complex submanifold and $\omega$ is the pullback of the Kähler form. Again, for any Hamiltonian circle action, every reducible level set is admissible.

Lemma 3.12. Let $M$ be a manifold with a circle action, $J$ an invariant complex structure, and $\mathcal{O}$ the induced orientation. Let $Z$ be an admissible hypersurface, and $X$ a connected component of the reduced space $M_{\mathrm{red}}=Z / S^{1}$. Then the reduced complex structure, $J_{\text {red }}$, is compatible with the reduced orientation, $\mathcal{O}_{\text {red }}$, on $X$ if and only if $\sigma_{J}(X)=1$.

Proof. By the definitions of the reduced complex structure, a positive frame on $M_{\text {red }}$ with respect to the complex orientation $J_{\text {red }}$ can be written as the push-forward of

$$
\eta_{1}, J \eta_{1}, \ldots, \eta_{d}, J \eta_{d}
$$

where $\left\{\eta_{i}\right\}$ is a complex basis for the maximal complex sub-bundle of $T Z$. The frame

$$
\eta_{1}, J \eta_{1}, \ldots, \eta_{d}, J \eta_{d}, \xi_{M}, J \xi_{M}
$$

on $M$ is positive with respect to the complex orientation. By the definition of the reduced orientation, the push-forward of (3.13) is positive with respect to $\mathcal{O}_{\text {red }}$ if and only if $\left(\xi_{M}, J \xi_{M}\right)$ is positive in $T \mathcal{S} \oplus N Z$.

3.5. Reduction of stable complex structures. Although an almost complex structure often fails to descend to the reduced space, it induces a stable complex structure, which does descend to the reduced space.

Let $M$ be a manifold with circle action, and let $Z$ be a reducible hypersurface. Take a stable complex structure $\mathcal{J}$ represented by a complex structure on $T M \oplus \mathbb{R}^{k}$. By (3.8), this complex structure restricts to an invariant complex structure on $\pi^{*}\left(T M_{\text {red }}\right) \oplus \mathbb{R}^{2} \oplus \mathbb{R}^{k}$, which in turn descends to a complex structure on $T M_{\text {red }} \oplus$ $\mathbb{R}^{2+k}$, providing a stable complex structure $\mathcal{J}_{\text {red }}$ on $M_{\text {red }}$. The reduced stable complex structure thus obtained will not depend on either the choice of isomorphism in (3.8) or the choice of representative of the equivariant stable complex structure.

Lemma 3.14. Let $S^{1}$ act on a manifold $M$, let $Z$ be an admissible hypersurface for an equivariant almost complex structure $J$, and let $\mathcal{J}=[J]$ denote the corresponding stable complex structure. Then $\mathcal{J}_{\text {red }}=\left[J_{\text {red }}\right]$.

Proof. Denote the maximal complex sub-bundle of $T Z$ by $\mathcal{V}$. As complex bundles, $i^{*} T M \cong \mathcal{V} \oplus \mathbb{C}$, where the trivial sub-line-bundle $\mathbb{C}$ inside the complex vector bundle $i^{*}(T M)$ has the trivializing section $\xi_{M}$. Therefore, $(\mathcal{V}, J)$ and $\left(i^{*} T M, J\right)$ are stably equivalent. The lemma follows.

3.6. Reduction of $\operatorname{spin}^{c}$ structures. Let $M$ be an oriented Riemannian manifold with a circle action. Let $(P, p)$ be an $S^{1}$-equivariant $\operatorname{spin}^{c}$ structure on $M$ with associated line bundle $L$. Let $i: Z \rightarrow M$ be the inclusion map of a reducible hypersurface $Z$ into $M$.

By equation (3.8), which reads

$$
i^{*}(T M) \cong \pi^{*}\left(T M_{\text {red }}\right) \oplus \mathbb{R}^{2},
$$

the invariant Riemannian metric on $M$ induces an invariant metric on $\pi^{*}\left(T M_{\text {red }}\right)$, hence a metric on $M_{\text {red }}$. The $\operatorname{spin}^{c}$ structure on $M$ restricts to an $S^{1}$-equivariant $\operatorname{spin}^{c}$ structure, $\left(i^{*} P, i^{*} p\right)$, on the vector bundle $i^{*} T M$. By (3.8) combined with Lemma 2.4, this induces an $S^{1}$-equivariant $\operatorname{spin}^{c}$ structure on $\pi^{*}\left(T M_{\text {red }}\right)$ with the 
same associated line bundle, $i^{*} L$. Taking the quotient by the $S^{1}$-action yields a $\operatorname{spin}^{c}$ structure on $M_{\text {red }}$ with associated line bundle $L_{\text {red }}$. This is independent of the choice of isomorphism in (3.8).

Remark 3.15. The reduction of a $\operatorname{spin}^{c}$ structure induced from a stable complex structure, a line bundle, an orientation, and a metric is isomorphic to the $\operatorname{spin}^{c}$ structure induced from the reduced stable complex structure, reduced line bundle, reduced orientation, and reduced metric.

3.7. Presymplectic reduction. Let $(M, \omega)$ be a presymplectic manifold with a circle action and moment map $\Phi: M \rightarrow \mathbb{R}$ (see Appendix A).

If $a$ is a regular value for $\Phi$, the circle action on the level set $\Phi^{-1}(a)$ is locally free. If this action is free, $\Phi^{-1}(a)$ is a reducible splitting hypersurface. Then there exists a unique presymplectic form $\omega_{\text {red }}^{a}$ on $M_{\text {red }}^{a}:=\Phi^{-1}(a) / S^{1}$ such that $\pi^{*} \omega_{\text {red }}=i^{*} \omega$.

Unlike in the symplectic case, the reduced space $M_{\text {red }}^{a}$ need not be connected even if $M$ is connected and $\Phi$ is proper.

If $[\omega+u \Phi]$ is the equivariant first Chern class of $L$ (see $\S 2.4$ ), then $\left[\omega_{\mathrm{red}}^{a}\right]$ is the first Chern class of $L_{\text {red }}^{a}$ if we reduce at $a=0$, but not otherwise.

3.8. Reduction and group actions. Let $M$ be a manifold with a circle action, and let $Z$ be a reducible hypersurface. Suppose that a second group, $G$, acts on $M$, and that this second action commutes with the circle action and preserves $Z$. Then we get a $G$-action on the reduced space, $M_{\text {red }}$. For each of the structures discussed in this section, if we begin with an $\left(S^{1} \times G\right)$-equivariant structure on $M$, reduction produces a $G$-equivariant structure on $M_{\text {red }}$.

\section{The MAin TheOREMS}

In this section we state three versions of "quantization commutes with reduction" corresponding to almost complex, stable complex, and $\operatorname{spin}^{c}$ quantizations. In each case, the theorem is written as an equality between virtual vector spaces, and holds when the quantization and reduction data are compatible in the following sense: if the weight by which $S^{1}$ acts on a fiber of the line bundle associated to the quantization data is large (small) over a fixed point, then that fixed point should be in $M^{+}\left(M^{-}\right)$. Finally, we prove that the first two of these theorems follow from the third.

Theorem 1. Almost complex quantization commutes with reduction, up to sign. Let the circle act on a compact manifold, $M$. Let $L$ be an equivariant line bundle over $M$, and let $J$ be an invariant almost complex structure on $M$. Let $Z \subset M$ be an admissible reducible hypersurface that splits $M$ into $M_{+}$and $M_{-}$(see Definitions 3.1, 3.3, and 3.9). Assume that the following conditions are satisfied for every component $F$ of the fixed-point set:

$$
\begin{aligned}
& \text { fiber weight at } F \geq-\sum \text { negative tangent weights at } F \quad \Rightarrow \quad F \subset M_{+}, \\
& \text {fiber weight at } F \leq-\sum \text { positive tangent weights at } F \Rightarrow F \subset M_{-},
\end{aligned}
$$

(see Remark 4.3). Then

$$
Q(M, L, J)^{S^{1}}=\sum_{X \subseteq M_{\mathrm{red}}} \sigma_{J}(X) \cdot Q\left(X, L_{\mathrm{red}}, J_{\mathrm{red}}\right),
$$


where the sum is taken over all connected components $X$ of the reduced space $M_{\mathrm{red}}=$ $Z / S^{1}, \sigma_{J}(X)$ is the sign associated to $X$ as in $(3.10), L_{\mathrm{red}}$ is the reduced line bundle (§3.3), $J_{\text {red }}$ is the reduced almost complex structure (§3.4), and $Q$ denotes almost complex quantization (\$2.1).

Remark 4.3. The fiber weight at $F$ is the weight by which $S^{1}$ acts on a fiber of $L$ over $F$. The tangent weights at $F$ are the weights by which $S^{1}$ acts on the complex vector space $\left(T_{m} M, J_{m}\right)$ for $m$ in $F$.

Remark 4.4. The sign $\sigma_{J}(X)$ in Theorem 1 comes from the discrepancy between the quotient orientation and the almost complex orientations. (See Lemma 3.12.)

Theorem 2. Stable complex quantization commutes with reduction. Let the circle act on a compact manifold $M$. Let $L$ be an equivariant line bundle over $M$, let $\mathcal{J}$ be an equivariant stable complex structure on $M$, and let $\mathcal{O}$ be an orientation on $M$. Let $Z$ be a reducible hypersurface that splits $M$ into $M_{+}$and $M_{-}$(see Definitions 3.1 and 3.3). Assume that the following conditions are satisfied for every component $F$ of the fixed-point set:

fiber weight at $F \geq-\sum$ negative tangent weights at $F \Rightarrow F \subset M_{+}$, fiber weight at $F \leq-\sum$ positive tangent weights at $F \Rightarrow F \subset M_{-}$,

(see Remark 4.7). Then

$$
Q(M, L, \mathcal{J}, \mathcal{O})^{S^{1}}=Q\left(M_{\text {red }}, L_{\text {red }}, \mathcal{J}_{\text {red }}, \mathcal{O}_{\text {red }}\right),
$$

where $M_{\mathrm{red}}=Z / S^{1}$ is the reduced space, $L_{\mathrm{red}}$ is the reduced line bundle (§3.3), $\mathcal{J}_{\text {red }}$ is the reduced stable complex structure (§3.5), $\mathcal{O}_{\text {red }}$ is the reduced orientation (§3.2), and $Q$ denotes stable complex quantization (§2.3).

Remark 4.7. Again, the fiber weight at $F$ means the weight by which $S^{1}$ acts on a fiber of $L$ over $F$. The negative and positive tangent weights of a stable complex $\mathcal{J}$ which is represented by a complex vector bundle $\left(T M \oplus \mathbb{R}^{k}, J\right)$, are the negative and positive weights by which $S^{1}$ acts on the complex vector space $\left(T_{m} M \oplus \mathbb{R}^{k}, J_{m}\right)$ for $m \in F$. These weights are well defined, even though different representatives of the stable complex structure may possess different numbers of zero weights.

We now state our "main theorem", which implies Theorems 1 and 2 . It will be proved in Section 7 .

Theorem 3. Main theorem: $\operatorname{spin}^{c}$ quantization commutes with reduction. Let the circle act on a compact oriented Riemannian manifold $M$. Let $(P, p)$ be an equivariant spin $^{c}$ structure on $M$. Let $Z$ be a reducible hypersurface that splits $M$ into $M_{+}$and $M_{-}$(see Definitions 3.1 and 3.3). Assume that the following conditions are satisfied for every component $F$ of the fixed-point set:

$$
\begin{aligned}
& \text { fiber weight at } F \geq \sum \mid \text { tangent weights at } F \mid \Rightarrow F \subset M_{+}, \\
& \text {fiber weight at } F \leq-\sum \mid \text { tangent weights at } F \mid \Rightarrow F \subset M_{-},
\end{aligned}
$$

(see Remark 4.10). Then

$$
Q(M, P, p)^{S^{1}}=Q\left(M_{\text {red }}, P_{\text {red }}, p_{\text {red }}\right),
$$

where $M_{\text {red }}=Z / S^{1}$ is the reduced space, $\left(P_{\mathrm{red}}, p_{\mathrm{red}}\right)$ is the reduced spin ${ }^{c}$ structure (§3.6), and $Q$ denotes spin ${ }^{c}$ quantization (§2.2). 
Remark 4.10. The fiber weight at $F$ means the weight by which $S^{1}$ acts on the fiber over $F$ of the line bundle associated to the $\operatorname{spin}^{c}$ structure. The tangent weights are defined only up to sign, because the vector space $T_{m} M$, for $m \in F$, is only a real representation space for $S^{1}$. However, their absolute values are well defined.

Proof that Theorem 1 follows from Theorem 2. We will now show that the almost complex version of "quantization commutes with reduction" follows from the stable complex version.

Let $M, L, J$, and $Z$ be as in the statement of Theorem 1 . Let $\mathcal{J}$ be the equivariant stable complex structure induced by $J$, and let $\mathcal{O}$ be the orientation induced by $J$. Conditions (4.1) and (4.5) are the same, because the nonzero tangent weights for $\mathcal{J}$ are the same as those for $J$. The corresponding quantizations are also the same: $Q(M, L, J)=Q(M, L, \mathcal{J}, \mathcal{O})$, and the almost complex quantization of the reduced space is equal to the stable complex quantization of the stable complex structure and orientation induced by $J_{\text {red }}$ (see Remark 2.9). The stable complex structure induced by $J_{\text {red }}$ coincides with $\mathcal{J}_{\text {red }}$ by Lemma 3.14 . However, on a component $X$ of $M_{\text {red }}$, the complex orientation of $J_{\text {red }}$ coincides with the reduced orientation $\mathcal{O}_{\text {red }}$ if and only if $\sigma_{J}(X)=+1$ (see Lemma 3.12). Since switching orientation results in switching the sign of the quantization, $Q\left(X, L_{\text {red }}, \mathcal{J}_{\text {red }}, \mathcal{O}_{\text {red }}\right)=\sigma_{J}(X) Q\left(X, L_{\text {red }}, J_{\text {red }}\right)$.

Proof that Theorem 2 follows from Theorem 3. We will now show that the stable complex version of "quantization commutes with reduction" follows from the $\operatorname{spin}^{c}$ version.

The data $(M, L, \mathcal{J}, \mathcal{O})$ (together with an invariant metric) determines an equivariant $\operatorname{spin}^{c}$ structure $(P, p)$ on $M$ (see $\left.\S 2.3\right)$.

First, we will show that conditions (4.5) for the stable complex structure imply conditions (4.8) for the associated $\operatorname{spin}^{c}$ structure. The line bundle associated to the $\operatorname{spin}^{c}$ structure is $L \otimes L \otimes K^{*}$, where $K^{*}$ is the dual to the canonical line bundle of the stable complex structure. Thus, over any component of the fixed point set,

the fiber weight of $L \otimes L \otimes K^{*}=2 \times$ (the fiber weight of $L$ )

$$
+\sum \text { the tangent weights, }
$$

and

$$
\begin{aligned}
\sum \mid \text { the tangent weights } \mid= & -2 \times \sum \text { the negative tangent weights } \\
& +\sum \text { the tangent weights. }
\end{aligned}
$$

Hence the first condition in (4.8), which reads

the fiber weight of $L \otimes L \otimes K^{*} \geq \sum \mid$ the tangent weights $\mid$,

is equivalent to the first condition in (4.5), which reads

the fiber weight of $L \geq-\sum$ the negative tangent weights.

A similar argument shows that the second conditions in (4.5) and (4.8) are equivalent.

Moreover, by the definition of the quantization of a stable complex structure, the corresponding equivariant quantizations are the same, $Q(M, L, \mathcal{J}, \mathcal{O})=Q(M, P, p)$, and the quantization of the $\operatorname{spin}^{c}$ structure induced by $\mathcal{J}_{\text {red }}$ and $\mathcal{O}_{\text {red }}$ is equal to 
the stable complex quantization of $\mathcal{J}_{\text {red }}$ and $\mathcal{O}_{\text {red }}$. Finally, the $\operatorname{spin}^{c}$ structure associated to $\left(M_{\text {red }}, L_{\text {red }}, \mathcal{J}_{\text {red }}, \mathcal{O}_{\text {red }}\right)$ (and the reduced metric) is isomorphic to the reduced $\operatorname{spin}^{c}$ structure, $\left(P_{\text {red }}, p_{\text {red }}\right)$.

Remark 4.11. The criteria which the hypersurface must satisfy at first seem surprisingly lax. However, this is much less surprising when one considers the following facts. Assume, for the sake of avoiding orbifolds, that the action of $S^{1}$ on $M$ is semi-free, that is, every point is fixed or free. Fix some quantizing information, and consider any splitting hypersurface $Z$. Let $\left\{F_{i}\right\}_{i=1}^{l}$ be the set of components of the fixed-point set contained in $M^{+}$. Let $B_{i}$ be a small tubular neighborhood of $F_{i}$, and let $S_{i}$ be its boundary. Then $Z / S^{1}$ is cobordant to $\coprod_{i=1}^{l} S_{i} / S^{1}$. (This cobordism was used by Victor Guillemin and by Shaun Martin.) Moreover, the cobording manifold, $\left(M \backslash \bigsqcup_{i} B_{i}\right) / S^{1}$, carries the quantization data. Since quantization is a cobordism invariant, this shows that $Q\left(Z / S^{1}\right)=\sum_{i=1}^{l} Q\left(S_{i} / S^{1}\right)$, and hence explains part of the freedom. The rest is explained once we notice that if $F_{i}$ is a component of the fixed-point set which is allowed to be in either $M^{+}$or $M^{-}$, the quantization of $S_{i} / S^{1}$ is zero.

Example 4.12. The four sphere, $S^{4}$, has no symplectic or almost complex structure (see $[\mathrm{BS}, \mathrm{K}]$ ). However, the inclusion

$j: S^{4} \hookrightarrow \mathbb{C}^{3}, \quad j\left(S^{4}\right)=\left\{\left(z_{1}, z_{2}, z_{3}\right):\left|z_{1}\right|^{2}+\left|z_{2}\right|^{2}+\left(\operatorname{Re} z_{3}\right)^{2}=1\right.$ and $\left.\operatorname{Im} z_{3}=0\right\}$, induces a stable complex structure,

$$
T S^{4} \oplus \mathbb{R}^{2} \cong j^{*}\left(T \mathbb{C}^{3}\right) \cong \mathbb{C}^{3},
$$

where the $\mathbb{R}^{2}$ is the trivial normal bundle of $S^{4}$ inside $\mathbb{C}^{3}$. Consider $S^{4}$ as a subset of $\mathbb{R}^{5} \cong\left\{\left(z_{1}, z_{2}, z_{3}\right): \operatorname{Im} z_{3}=0\right\}$, and orient it so that when taken together with the outward normal, it gives the standard orientation on $\mathbb{R}^{5}$.

Let $L=\mathbb{C}$ be the trivial line bundle over $S^{4}$ equipped with the following $S^{1}$ action:

$$
e^{i \theta} \cdot\left(\left(z_{1}, z_{2}, z_{3}\right), v\right)=\left(\left(e^{i \theta} z_{1}, e^{i \theta} z_{2}, z_{3}\right), e^{m i \theta} v\right)
$$

for $\left(z_{1}, z_{2}, z_{3}\right) \in S^{4}$ and $v \in \mathbb{C}$. The equator, $Z=\left\{\left(z_{1}, z_{2}, 0\right):\left|z_{1}\right|^{2}+\left|z_{2}\right|^{2}=\right.$ $1\} \cong S^{3}$, is a reducible hypersurface that splits $M$ into the northern and southern hemispheres. At each of the two fixed points, $N=(0,0,1)$ and $S=(0,0,-1)$, the non-zero weights of the stable tangent bundle are +1 and +1 , and the fiber weight of $L$ is $m$. Therefore, the conditions of Theorem 2 are satisfied exactly when $m=-1$.

We can now check by computing explicitly, that the orientation on $S^{4}$ agrees with that of $\mathbb{C}^{2} \cong\left\{\left(z_{1}, z_{2}, 0\right) \in \mathbb{C}^{3}\right\}$ at $N$, but is opposite at $S$. Therefore, the two fixed-point contributions to the index-character formula cancel each other. Hence, $\operatorname{dim} Q(M)^{S^{1}}=0$ for all $m$. On the other hand, $M_{\text {red }}=S^{2}, \mathcal{J}_{\text {red }}$ is the class of the standard complex structure, $\mathcal{O}_{\text {red }}$ is also standard, and $L_{\text {red }}=\mathcal{O}(m)$. Consequently, $\operatorname{dim} Q\left(M_{\text {red }}\right)=m+1$. As required, $Q(M)^{S^{1}}=Q\left(M_{\text {red }}\right)$ when $m=-1$.

Example 4.13. Let $S^{1}$ act on any spin manifold $M$, and consider the $\operatorname{spin}^{c}$ bundle with trivial associated line bundle. The criteria are automatically satisfied on every fixed point set, so quantization commutes with reduction for every splitting hypersurface. In this case, both are trivial.

Example 4.14. Notice that our theorem makes sense whenever $Z$ is a reducible hypersurface. However, even if $M \backslash Z$ can be written as the disjoint union of an $M^{-}$ 
and an $M^{+}$which satisfy conditions (4.1), (4.5), or (4.8), the theorem is generally not true unless $Z$ is splitting.

Consider the torus $T=\mathbb{R}^{2} / \mathbb{Z}^{2}$ with the complex structure induced by the isomorphism $\mathbb{R}^{2}=\mathbb{C}$ and the trivial line bundle. The circle $\mathbb{R} / \mathbb{Z}$ acts on $T$ by $[\alpha] \cdot[x, y]=[x+\alpha, y]$.

Because there are no fixed points, by Atiyah-Segal-Singer the almost complex quantization of $T$ is the zero representation. In particular, the zero weight space is zero.

On the other hand, the circle $Z:=[x, 0]$ is a reducible hypersurface. If we take $M^{+}:=T \backslash Z$ and $M^{-}=\emptyset$, then they satisfy conditions (4.1) since neither contains any fixed point. Nevertheless, the reduced space is a point, so its quantization is not zero. Quantization does not commute with reduction in this example, because $Z$ is not splitting.

\section{Presymplectic COROllaries}

The most important application of our theorems is to presymplectic manifolds, where we quantize and reduce with respect to the same closed two form and moment map. In this case, the compatibility conditions in the earlier theorems are automatically satisfied, and the appropriate version of "quantization commutes with reduction" holds at every regular integer value.

More precisely, let $S^{1}$ act on a manifold $M$. Let $\omega$ be a presymplectic form, that is, a closed invariant two-form with a moment map $\Phi: M \rightarrow \mathbb{R}$. Assume that $\omega+u \Phi$ is prequantizable, i.e., that either $\omega+u \Phi$ is complex prequantizable and that $M$ has an invariant almost complex structure or an invariant stable complex structure and an orientation, or that $M$ is oriented and $\omega+u \Phi$ is $\operatorname{spin}^{c}$ prequantizable (see $\S 2.4)$. If zero is a regular value of $\Phi$, then $Z=\Phi^{-1}(0)$ is automatically a reducible splitting hypersurface. Moreover, the compatibility conditions - conditions (4.1), (4.5), or (4.8) - are automatically satisfied: the fiber weight at $F$ is equal to either $\Phi(F)$ (in the almost complex and stable complex cases) or $2 \Phi(F)$ (in the $\operatorname{spin}^{c}$ case); this is positive if $F$ is in $M_{+}$and negative if $F$ is in $M_{-}$. The compatibility conditions follow immediately by comparing the signs of the left and right terms in the inequalities (4.1), (4.5), or (4.8). This shows that quantization commutes with reduction at zero.

More generally, consider any regular $a \in \mathbb{Z}$. Then the reduction $\omega_{\text {red }}^{a}$ of $[\omega+u \Phi]$ at $a \in \mathbb{R}$ is identical to the reduction of $[\omega+u(\Phi-a)]$ at 0 . Therefore, by the preceding paragraph, the multiplicity of 0 in $Q(M, \omega, \Phi-a, \ldots)$ is $\operatorname{dim} Q\left(M_{\text {red }}^{a}, \omega_{\text {red }}^{a}, \ldots\right)$. But, by Remark 2.13, the multiplicity of 0 in $Q(M, \omega, \Phi-a, \ldots)$ is simply the multiplicity of $a$ in $Q(M, \omega, \Phi, \ldots)$. Theorems 1-3 thus have the following corollaries.

Corollary 1. Almost complex quantization of presymplectic manifolds commutes with reduction, up to sign. Let $S^{1}$ act on a compact presymplectic and almost complex manifold $(M, \omega, J)$ with a moment map $\Phi$. Assume that $\omega$ and $\Phi$ are integral (see §2.4). Let $a \in \mathbb{Z}$ be a regular value for $\Phi$ such that the $S^{1}$ action on the a-level set is free and the a-level set is admissible (see Definition 3.9). Then

$$
Q(M, \omega, \Phi, J)^{a}=\sum_{X \subseteq M_{\mathrm{red}}^{a}} \sigma_{J}(X) \cdot Q\left(X, \omega_{\mathrm{red}}^{a}, J_{\text {red }}^{a}\right),
$$

where we sum over the connected components $X$ of the reduced space $M_{\mathrm{red}}^{a}:=$ $\Phi^{-1}(a) / S^{1}, \sigma_{J}(X)= \pm 1$ is the sign associated to $X$ as in (3.10), $\omega_{\text {red }}^{a}$ is the reduced 
presymplectic form (§3.7), $J_{\text {red }}^{a}$ is the reduced almost complex structure (§3.4), $Q$ denotes the almost complex quantization (§2.4), and $Q(\cdot)^{a}$ is the ath weight space in $Q(\cdot)$.

Remark 5.2. When $\omega$ is symplectic and $J$ is a compatible almost complex structure, every reducible hypersurface is admissible, and $\sigma_{J}\left(M_{\text {red }}^{a}\right)=1$. In this case, Corollary 1 reduces to the usual theorem, that "quantization commutes with reduction" for almost Kähler quantization on symplectic manifolds.

Corollary 2. Stable complex quantization of presymplectic manifolds commutes with reduction. Let $S^{1}$ act on a compact oriented presymplectic and stable complex manifold $(M, \omega, \mathcal{J}, \mathcal{O})$ with a moment map $\Phi$. Assume that $\omega$ and $\Phi$ are integral (see §2.4). Assume that $a \in \mathbb{Z}$ is a regular value for $\Phi$ and that the $S^{1}$ action on the a-level set is free. Let $\mathcal{J}$ be an equivariant stable complex structure. Then

$$
Q(M, \omega, \Phi, \mathcal{J}, \mathcal{O})^{a}=Q\left(M_{\text {red }}^{a}, \omega_{\text {red }}^{a}, \mathcal{J}_{\text {red }}^{a}, \mathcal{O}_{\text {red }}^{a}\right),
$$

where $M_{\mathrm{red}}^{a}:=\Phi^{-1}(a) / S^{1}$ is the reduced space, $\omega_{\mathrm{red}}^{a}$ is the reduced presymplectic form (§3.7), $\mathcal{O}_{\text {red }}^{a}$ is the reduced orientation (§3.2), $\mathcal{J}_{\text {red }}^{a}$ is the reduced stable complex structure (§3.5), $Q$ denotes the stable complex quantization (§2.3 and §2.4), and $Q(\cdot)^{a}$ is the ath weight space in $Q(\cdot)$.

Corollary 3. Spin ${ }^{c}$ quantization of presymplectic manifolds commutes with reduction. Let $S^{1}$ act on a compact presymplectic manifold, $(M, \omega)$, with a moment map $\Phi$. Assume that $(M, \omega, \Phi)$ is equivariantly spin ${ }^{c}$ prequantizable (see §2.4). Assume that $a \in \mathbb{Z}$ is a regular value for $\Phi$ and that the $S^{1}$ action on the a-level set is free. Let $\mathcal{O}$ be an orientation on $M$. Then

$$
Q(M, \omega, \Phi, \mathcal{O})^{S^{1}}=Q\left(M_{\text {red }}^{a}, \omega_{\text {red }}^{a}, \mathcal{O}_{\text {red }}^{a}\right),
$$

where $M_{\mathrm{red}}^{a}=\Phi^{-1}(a) / S^{1}$ is the reduced space, $\omega_{\mathrm{red}}^{a}$ is the reduced presymplectic form (§3.7), $\mathcal{O}_{\text {red }}^{a}$ is the reduced orientation (§3.2), $Q$ denotes the spin ${ }^{c}$ quantization (§2.2 and §2.4), and $Q(\cdot)^{a}$ is the ath weight space in $Q(\cdot)$.

Remark 5.5. Assume that 0 is a regular value of $\Phi$. Since tangent weights are always integers, conditions (4.1) also hold for $\Phi^{-1}(t)$ for all $-1<t<1$. Thus, as long as $t$ is a regular value such that the $S^{1}$ action on the $t$ level set is free and the $t$ level set is admissible, formula (4.2) holds for $M_{\text {red }}^{t}=\Phi^{-1}(t) / S^{1}$. (This does not follow from Corollary 1.) A similar result holds in the stable complex case.

A much stronger, and more useful, result holds in the $\operatorname{spin}^{c}$ case: even if 0 is a singular value, conditions (4.8) hold for $Z=\Phi^{-1}(t)$ for all $-\frac{1}{2}<t<\frac{1}{2}$. (The boundaries are $\pm \frac{1}{2}$ and not \pm 1 because the fiber weight at $F$ is $2 \Phi(F)$, not $\Phi(F)$; see $\S 2.4$.) Thus formula (4.9) holds for any $M_{\text {red }}^{t}=\Phi^{-1}(t) / S^{1}$ with $-\frac{1}{2}<t<\frac{1}{2}$. The manifold $M_{\text {red }}^{t}$ then provides a "resolution" of $\Phi^{-1}(0) / S^{1}$, if the latter is singular, in the spirit of Meinrenken and Sjamaar [MS]. Again, this does not follow from Corollary 3.

Remark 5.6. Let $S^{1}$ act on an almost complex manifold $(M, J)$. Let $L$ be a complex Hermitian line bundle, and let $K^{*}$ be the dual of the canonical line bundle. Let $\omega$ and $\alpha$ be two-forms representing the first Chern class of $L$ and $K^{*}$, respectively. Let $\Phi$ and $\Psi$ be their respective moment maps. Consider the moment map

$$
\Phi_{t}=\Phi+t \Psi
$$


for the presymplectic form

$$
\omega_{t}=\omega+t \alpha
$$

Then for all $t \in[0,1]$, if $\Phi_{t}^{-1}(0)$ is admissible, formula (4.2) still holds for

$$
M_{\mathrm{red}}^{t}=\Phi_{t}^{-1}(0) / S^{1} \text {. }
$$

This follows from Theorem 1. The compatibility conditions, (4.1), are automatically satisfied:

$$
\begin{aligned}
F \subset M_{+} & \Leftrightarrow \Phi_{t}(F)>0 \\
& \Leftrightarrow \text { fiber weight at } F+t \sum \text { tangent weights at } F>0 \\
& \Rightarrow \text { fiber weight at } F>-t \sum \text { tangent weights at } F \\
& \geq-t \sum \text { positive tangent weights at } F \\
& \geq-\sum \text { positive tangent weights at } F
\end{aligned}
$$

and, similarly,

$$
F \subset M_{-} \Rightarrow \text { fiber weight at } F<-\sum \text { negative tangent weights at } F \text {. }
$$

Corollary 1 is the case $t=0$, and Corollary 3 (for the associated $\operatorname{spin}^{c}$ structure) corresponds to the middle point, $t=\frac{1}{2}$. Hence, Corollary 3 does not imply Corollary 1; they are different theorems. A similar remark holds for stable complex quantization.

Example 5.7. Let $S^{1}$ rotate $S^{2}$, fixing the north and south poles. Consider the two-form $m \sigma$, where $m$ is an integer, and $\sigma$ is the standard Kähler form on $S^{2} \simeq \mathbb{C P}^{1}$ with total integral equal to one. Choose the moment map $\Phi: S^{2} \rightarrow \mathbb{R}$ so that it takes values 0 and $m$ at the south and north poles, respectively. Equip $S^{2}$ with the standard complex structure, $J$.

The line bundle with equivariant first Chern class $[\omega+u \Phi]$ is $\mathcal{O}(m)$ with fiber weights 0 and $m$ over the south and north poles, respectively.

If $0<m$, the multiplicity of $a$ in the quantization, $Q\left(S^{2}, m \sigma, \Phi, J\right)$, is 1 for all integers $a$ such that $0 \leq a \leq m$, and is 0 otherwise. The singular values are $a=0, m$. For $0<a<m, M_{\text {red }}^{a}$ is a single point with reduced orientation +1 . If $a<0$ or $a>m, M_{\text {red }}^{a}$ is empty. Hence for any regular integer $a, Q\left(S^{2}\right)^{a}=Q\left(M_{\text {red }}^{a}\right)$; the dimension of these spaces is +1 if $0<a<m$ and 0 if $a<0$ or $a>m$.

If $m<0$, the multiplicity of $a$ in the quantization $Q\left(S^{2}, m \sigma, \Phi, J\right)$ is -1 for all integers $a$ such that $m<a<0$, and is 0 otherwise. The singular values are $a=0, m$. For $m<a<0, M_{\text {red }}^{a}$ is a single point with reduced orientation -1 . If $a<m$ or $a>0, M_{\text {red }}^{a}$ is empty. Hence for any regular integer $a, Q\left(S^{2}\right)^{a}=-Q\left(M_{\text {red }}^{a}\right)$; the dimension of these spaces is -1 if $m<a<0$ and 0 if $a<m$ or $a>0$.

Example 5.8. Let $S^{1}$ rotate $S^{2}$ and consider the two-form $m \sigma$, as in the previous example. Choose the moment map $\Phi: S^{2} \rightarrow \mathbb{R}$ so that it takes values $-1 / 2$ and $m-1 / 2$ at the south and north poles, respectively.

If $0<m$, the multiplicity of $a$ in the $\operatorname{spin}^{c}$ quantization, $Q\left(S^{2}, m \sigma, \Phi, \mathcal{O}\right)$, is +1 for all integers $a$ such that $0 \leq a<m$ and 0 otherwise. The singular values are $a=-1 / 2, m-1 / 2$. For $-1 / 2<a<m-1 / 2, M_{\text {red }}$ is a single point with reduced orientation +1 . For $a<-1 / 2$ or $a>m-1 / 2, M_{\text {red }}$ is empty. Hence $Q\left(S^{2}\right)^{a}=Q\left(M_{\text {red }}^{a}\right)$ for all integers $a$; the dimensions of these spaces are +1 if $0 \leq a<m$ and 0 otherwise. 
If $m<0$, then the multiplicity of $a$ in the quantization $Q\left(S^{2}, m \sigma, \Phi, \mathcal{O}\right)$ is -1 for all integers $a$ such that $m \leq a<0$, and 0 otherwise. The singular values are $a=-1 / 2$ and $a=m-1 / 2$. For $m-1 / 2<a<-1 / 2, M_{\text {red }}^{a}$ is a single point, with reduced orientation -1 . For $a<m-1 / 2$ or $a>-1 / 2, M_{\text {red }}^{a}$ is empty. Hence $Q\left(S^{2}\right)^{a}=Q\left(M_{\mathrm{red}}^{a}\right)$ for all integers $a$; the dimensions of these spaces are -1 if $m \leq a<0$ and 0 otherwise.

Example 5.9. Now consider the presymplectic form $1 / 2 \pi(10 z+5) d z d \theta$ with moment map $\Phi(z, \theta)=5 z^{2}+5 z+1$, where $(z, \theta)$ are cylindrical coordinates on $S^{2}$, the unit sphere in $\mathbb{R}^{3}$.

We will work out the almost complex and the stable complex quantizations. The quantizing line bundle is $O(10)$, with fiber weight 11 at the north pole and 1 at the south pole. The multiplicity of the weight $a$ is +1 if $1 \leq a \leq 11$ and 0 otherwise.

The zero-level set has two components, hence so does the reduced space: $M_{\text {red }}=$ $X_{1} \sqcup X_{2}$. Almost complex quantization gives $\operatorname{dim} Q\left(X_{1}\right)=\operatorname{dim} Q\left(X_{2}\right)=+1$. However, $\sigma_{J}\left(X_{1}\right)=+1$ and $\sigma_{J}\left(X_{2}\right)=-1$, so Corollary 1 gives $\operatorname{dim} Q\left(S^{2}\right)^{S^{1}}=$ $\operatorname{dim} Q\left(X_{1}\right)-\operatorname{dim} Q\left(X_{2}\right)=0$.

Stable complex quantization for the stable complex structures associated to these almost complex structures is very similar. The only difference lies in the definition of the reduced orientation. It gives $\operatorname{dim} Q\left(S^{2}\right)^{S^{1}}=\operatorname{dim} Q\left(X_{1}\right)+\operatorname{dim} Q\left(X_{2}\right)$, with $\operatorname{dim} Q\left(X_{1}\right)=+1$ and $\operatorname{dim} Q\left(X_{2}\right)=-1$.

\section{The CUT SPACE}

Following [DGMW], to prove that quantization commutes with reduction we construct a new space by cutting. The technique of symplectic cutting was introduced by Lerman in [L] for symplectic manifolds with a circle action and a moment map. We apply Lerman's construction to manifolds with other structures. Our goal in this section is to prove the following proposition.

Proposition 6.1. Let the circle group, $S^{1}$, act on a smooth manifold $M$. Let $Z$ be a reducible hypersurface (see Definitions 3.1 and 3.3) that splits $M$ into $M_{+}$and $M_{-}$, and let $M_{\mathrm{red}}:=Z / S^{1}$ be the reduced space.

1. There exists a smooth manifold, $M_{\mathrm{cut}}$, with a circle action, and equivariant embeddings

$$
i_{+}: M_{+} \hookrightarrow M_{\mathrm{cut}} \quad \text { and } \quad i_{\mathrm{red}}: M_{\mathrm{red}} \hookrightarrow M_{\mathrm{cut}},
$$

such that $M_{\text {cut }}$ is the disjoint union of $i_{+}\left(M_{+}\right)$and $i_{\text {red }}\left(M_{\text {red }}\right)$. Here the circle action on $M_{+}$is induced from $M$, and the action on $M_{\text {red }}$ is trivial.

2. The normal bundle to the submanifold $i_{\mathrm{red}}\left(M_{\mathrm{red}}\right)$ in $M_{\mathrm{cut}}$, pulled back to $M_{\mathrm{red}}$ via the map $i_{\mathrm{red}}$, is isomorphic to the bundle

$$
N:=Z \times{ }_{S^{1}} \mathbb{C}
$$

over $M_{\mathrm{red}}:=Z / S^{1}$. Here $[m, z]=\left[a \cdot m, a^{-1} z\right]$ for all $a \in S^{1}$, and the circle acts on this bundle, $N$, by $\lambda \cdot[m, z]=[m, \lambda z]$.

3. Given an orientation on $M$, there exists an orientation on $M_{\text {cut }}$ such that

(a) the open embedding $i_{+}: M_{+} \hookrightarrow M_{\text {cut }}$ preserves orientation;

(b) the orientation of the submanifold $i_{\text {red }}\left(M_{\text {red }}\right)$ coming from the reduced orientation of $M_{\mathrm{red}}$ (see §3.2), followed by the orientation on the normal bundle of $i_{\mathrm{red}}\left(M_{\mathrm{red}}\right)$ that is induced from the complex orientation on the fibers of (6.2), gives the orientation on $M_{\mathrm{cut}}$. 
4. Let $(P, p)$ be an equivariant spin ${ }^{c}$ structure over $M$ with an associated line bundle $L$. Then there exists an equivariant $\operatorname{spin}^{c}$ structure $\left(P_{\text {cut }}, p_{\text {cut }}\right)$ on $M_{\text {cut }}$ whose associated line bundle, $L_{\text {cut }}$, pulls back to the following $S^{1}$-equivariant complex line bundles on $M_{+}$and on $M_{\text {red }}$ :

(a) $\left.i_{+}^{*} L_{\text {cut }} \cong L\right|_{M_{+}}$

(b) $i_{\text {red }}^{*} L_{\text {cut }} \cong L_{\text {red }} \otimes N$ (see $§ 3.3$ and (6.2)).

Proof of part 1. We can assume that $Z$ is the zero level set of an invariant function $\Phi: M \rightarrow \mathbb{R}$ for which 0 is a regular value, and that $M_{+}$is the pre-image under $\Phi$ of the positive numbers. Consider the product $M \times \mathbb{C}$ with the anti-diagonal circle action, $a \cdot(m, z)=\left(a \cdot m, a^{-1} z\right)$, and with the function $\tilde{\Phi}(m, z):=|z|^{2}-\Phi(m)$. It is easy to check that 0 is a regular value for $\tilde{\Phi}$ and that $S^{1}$ acts freely on the zero level set of $\tilde{\Phi}$,

$$
\tilde{Z}=\left\{\left.(m, z)|\Phi(m)=| z\right|^{2}\right\} .
$$

The cut space, defined by

$$
M_{\text {cut }}:=\tilde{Z} / S^{1}=(M \times \mathbb{C})_{\mathrm{red}},
$$

is therefore a smooth manifold. Let $S^{1}$ act on $M_{\text {cut }}$ by $\lambda \cdot[m, z]=[\lambda \cdot m, z]=[m, \lambda z]$.

The inclusion map $i_{+}: M_{+} \hookrightarrow M_{\text {cut }}$ is induced by the following commuting diagram:

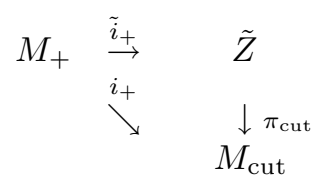

from the map $\tilde{i}_{+}(m):=(m, \sqrt{\Phi(m)})$. The inclusion map $i_{\text {red }}: M_{\text {red }} \hookrightarrow M_{\text {cut }}$ is induced by the following commuting diagram:

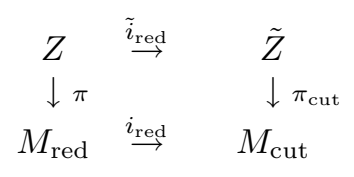

from the map $\tilde{i}_{\text {red }}(m):=(m, 0)$.

Proof of part 2. The submanifold $i_{\text {red }}\left(M_{\text {red }}\right)$ of $M_{\text {cut }}$ is the quotient of the submanifold $\tilde{i}_{\text {red }}(Z)=Z \times\{0\}$ of $\tilde{Z}$. The normal bundle of the latter is $Z \times \mathbb{C}$, with the anti-diagonal circle action induced from $M \times \mathbb{C}$.

Proof of part 3(a). The orientations on $M$ and $\mathbb{C}$ induce an orientation on $M \times \mathbb{C}$ which, by $\S 3.2$, descends to an orientation on $M_{\text {cut }}=(M \times \mathbb{C})_{\text {red }}$. Recall a frame on $M_{\text {cut }}$ is oriented if and only if its lifting to $\tilde{Z}$, followed by the generator of the anti-diagonal circle action on $\tilde{Z}$, and further followed by a positive normal to $\tilde{Z}$, gives an oriented frame on $M \times \mathbb{C}$.

Let $\eta_{1}, \ldots, \eta_{d}$ be an oriented frame on $M_{+}$. Its push-forward to $\tilde{Z}$ has the form

$$
\left(\tilde{i}_{+}\right)_{*} \eta_{i}=\eta_{i}+a_{i} \frac{\partial}{\partial r}
$$

for some real numbers $a_{i}$. (On the right we identify the vectors $\eta_{i} \in T_{m} M$ and $\frac{\partial}{\partial r} \in T_{z} \mathbb{C}$ with their images in $T_{(m, z)}(M \times \mathbb{C}) \simeq T_{m} M \times T_{z} \mathbb{C}$.) The map $i_{+}$ 
preserves orientation if and only if the following frame on $M \times \mathbb{C}$ is oriented:

$$
\eta_{1}+a_{1} \frac{\partial}{\partial r}, \ldots, \eta_{d}+a_{d} \frac{\partial}{\partial r}, \xi_{M}-\frac{\partial}{\partial \theta}, \frac{\partial}{\partial r}
$$

where $r, \theta$ are polar coordinates on $\mathbb{C}$; this is because $\xi_{M}-\frac{\partial}{\partial \theta}$ generates the antidiagonal circle action, and $\frac{\partial}{\partial r}$ is a positive normal to $\tilde{Z}$. Finally, it is easy to check that (6.5) is an oriented frame on $M \times \mathbb{C}$.

Proof of part 3(b). Fix a point $m$ in $Z$. Consider an oriented basis for $T_{m} M$ of the form

$$
\zeta_{1}, \ldots, \zeta_{d}, \xi_{M}, n
$$

where $\zeta_{i}$ are tangent to $T_{m} Z$, where $\xi_{M}$ generates the circle action, and where $n$ is a positive normal vector to $Z$ in $M$. By definition of the reduced orientation, the vectors $\zeta_{1}, \ldots, \zeta_{d}$ descend to an oriented frame on $M_{\text {red }}$.

The vectors

$$
\zeta_{1}, \ldots, \zeta_{d}, \frac{\partial}{\partial x}, \frac{\partial}{\partial y}, \xi_{M}, n
$$

form an oriented basis of $T_{(m, 0)}(M \times \mathbb{C})$, where $x, y$ are the Euclidean coordinates on $\mathbb{C}$. (Here we identified the vectors $\zeta_{i}, \xi_{M}, n \in T_{m} M$ and $\frac{\partial}{\partial x}, \frac{\partial}{\partial y} \in T_{0} \mathbb{C}$ with their images in $T_{(m, 0)}(M \times \mathbb{C})=T_{m} M \times T_{0} \mathbb{C}$.) Again by definition of the reduced orientation, the vectors

$$
\zeta_{1}, \ldots, \zeta_{d}, \frac{\partial}{\partial x}, \frac{\partial}{\partial y}
$$

descend to an oriented frame on $M_{\text {cut }}$. Since the first $d$ of these descend to an oriented frame on the submanifold $i_{\text {red }}\left(M_{\text {red }}\right)$, and the last two descend to an oriented basis of the fiber of the normal bundle when identified with $\mathbb{C}$, we conclude that the orientation of the submanifold $i_{\text {red }}\left(M_{\text {red }}\right)$ followed by that of its normal bundle gives the orientation of $M_{\text {cut }}$.

Proof of part 4 . We will now describe a $\operatorname{spin}^{c}$ structure on $M_{\text {cut }}$. First, noting that $\operatorname{spin}^{c}(2)=U(1) \times_{\mathbb{Z}_{2}} U(1)$, we define an equivariant $\operatorname{spin}^{c}$ structure over $\mathbb{C}$ by the trivial principal bundle

$$
\mathbb{C} \times\left(U(1) \times_{\mathbb{Z}_{2}} U(1)\right) \rightarrow \mathbb{C}
$$

with the non-trivial circle action

$$
e^{i \theta} \cdot(z,[a, b])=\left(e^{i \theta} z,\left[e^{i \theta / 2} a, e^{-i \theta / 2} b\right]\right)
$$

The associated line bundle is the trivial line bundle $C$ over $\mathbb{C}$ with the circle action

$$
e^{i \theta} \cdot(z, b)=\left(e^{i \theta} z, e^{-i \theta} b\right) .
$$

In Lemma 6.10 below we define a $\operatorname{spin}^{c}$ structure on the product of any two spin ${ }^{c}$ manifolds. In this way we get a $\operatorname{spin}^{c}$ structure on $M \times \mathbb{C}$, which in turn descends to a $\operatorname{spin}^{c}$ structure on $M_{\text {cut }}=(M \times \mathbb{C})_{\text {red }}$ (see $\left.\S 3.6\right)$.

The associated line bundle of the $\operatorname{spin}^{c}$ structure on $M \times \mathbb{C}$ is $\operatorname{pr}_{1}^{*} L \otimes \operatorname{pr}_{2}^{*} C$, where $\mathrm{pr}_{1}, \mathrm{pr}_{2}$ are the projections from $M \times \mathbb{C}$ to $M$ and to $\mathbb{C}$, respectively. Again by $\S 3.6$, the line bundle associated to the $\operatorname{spin}^{c}$ structure $M_{\text {cut }}$ is the reduced line bundle,

$$
L_{\text {cut }}=\left(\operatorname{pr}_{1}^{*} L \otimes \operatorname{pr}_{2}^{*} C\right)_{\text {red }}
$$

reduced with respect to the anti-diagonal circle action. On it we have a left circle action induced from the given action on $L$ and the trivial action on $\operatorname{pr}_{2}^{*} C$. 
Over $M_{+}$we have

$$
i_{+}^{*} L_{\text {cut }}=\tilde{i}_{+}^{*}\left(\operatorname{pr}_{1}^{*} L \otimes \operatorname{pr}_{2}^{*} C\right)=L \otimes \mathbb{C}=L
$$

as $S^{1}$-equivariant complex line bundles, because $\operatorname{pr}_{1} \circ \tilde{i}_{+}=\operatorname{id}_{M_{+}}$, and because the left circle action is trivial on the $\mathbb{C}$ piece. This proves part $4(\mathrm{a})$.

Over $Z$ we have

$$
\pi^{*} i_{\text {red }}^{*} L_{\text {cut }}=\tilde{i}_{\text {red }}^{*}\left(\operatorname{pr}_{1}^{*} L \otimes \operatorname{pr}_{2}^{*} C\right)=i^{*} L \otimes \mathbb{C}
$$

where $i$ is the inclusion of $Z$ into $M$, and where $\mathbb{C}$ is the trivial complex line bundle over $Z$ with the anti-standard circle action. In particular, $\mathbb{C}_{\text {red }}=Z \times_{S^{1}} \mathbb{C}=N$, hence

$$
i_{\text {red }}^{*} L_{\text {cut }}=\left(i^{*} L \otimes \mathbb{C}\right)_{\text {red }}=L_{\text {red }} \otimes \mathbb{C}_{\text {red }}=L_{\text {red }} \otimes N
$$

This proves part $4(\mathrm{~b})$, thus completing the proof of Proposition 6.1.

In the course of the above proof we used the following lemma:

Lemma 6.10. The product of two equivariant spinc manifolds has a natural equivariant $\operatorname{spin}^{c}$ structure, whose associated line bundle is the exterior tensor product of the two original associated line bundles.

Proof. The inclusion $j: \mathrm{SO}(m) \times \mathrm{SO}(n) \hookrightarrow \mathrm{SO}(m+n)$ lifts to an inclusion $j$ : $\operatorname{spin}(m) \times_{\mathbb{Z}_{2}} \operatorname{spin}(n) \hookrightarrow \operatorname{spin}(m+n)$. Define a group homomorphism $j: \operatorname{spin}^{c}(m) \times$ $\operatorname{spin}^{c}(n) \rightarrow \operatorname{spin}^{c}(m+n)$ by

$$
j([A, a],[B, b])=[j(A, B), a b]
$$

(where square brackets denote $\mathbb{Z}_{2}$-equivalence class in $\operatorname{spin}^{c}(k)=\operatorname{spin}(k) \times_{\mathbb{Z}_{2}} U(1)$ ). The following diagrams commute:

$$
\begin{aligned}
& \operatorname{spin}^{c}(m) \times \operatorname{spin}^{c}(n) \stackrel{j}{\hookrightarrow} \operatorname{spin}^{c}(m+n) \\
& f \times f \downarrow \quad f \downarrow \\
& \mathrm{SO}(m) \times \mathrm{SO}(n) \quad \stackrel{j}{\hookrightarrow} \quad \mathrm{SO}(m+n)
\end{aligned}
$$

and

$$
\begin{array}{cccc}
\operatorname{spin}^{c}(m) \times \operatorname{spin}^{c}(n) & \stackrel{j}{\hookrightarrow} & \operatorname{spin}^{c}(m+n) \\
\operatorname{det} \times \operatorname{det} \downarrow & & \operatorname{det} \downarrow \\
U(1) \times U(1) & \stackrel{\times}{\longrightarrow} & U(1)
\end{array}
$$

where the bottom arrow is multiplication, and where $f$ and det are as in $\S 2.2$.

Let $(M, P, p)$ and $\left(M^{\prime}, P^{\prime}, p^{\prime}\right)$ be $\operatorname{spin}^{c}$ manifolds. Let $\tilde{P}$ denote the $\operatorname{spin}^{c}(n+m)$ bundle over $M \times M^{\prime}$ that is associated to the bundle $P \times P^{\prime}$ by $j: \operatorname{spin}^{c}(n) \times$ $\operatorname{spin}^{c}(m) \rightarrow \operatorname{spin}^{c}(n+m)$. By diagram (6.11) it is clear that $p \times p^{\prime}$ naturally

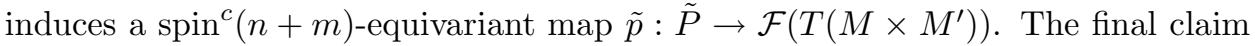
follows from diagram (6.12).

\section{Proof OF THE MAIN THEOREM}

We are now ready to prove our main theorem, Theorem 3, that "spinc quantization commutes with reduction". Following [DGMW], we prove this theorem by applying the Atiyah-Segal-Singer index theorem to the manifold $M$ and to the cut space $M_{\text {cut }}$, and then compare the results. 
Let the circle group act on a compact manifold $M$. Let $(P, p)$ be an equivariant $\operatorname{spin}^{c}$ structure on $M$ with an associated line bundle $L$. By the Atiyah-Segal-Singer index theorem [ASIII], the character, $\chi$, (defined in $\S 1$ ) of the equivariant index of the $\operatorname{spin}^{c}$-Dirac operator is given by a sum over the connected components of the fixed point set:

$$
\chi(\exp (u))=\sum_{F} A S_{F}(u), \quad u \in \operatorname{Lie}\left(S^{1}\right) .
$$

The contribution of the fixed component $F$ is

$$
\operatorname{AS}_{F}(u)=(-1)^{m} \int_{F} \exp \left(\frac{1}{2} \tilde{c}_{1}\left(\left.L\right|_{F}\right)\right) \hat{A}(F) \frac{\hat{A}(N F)}{\operatorname{Euler}(N F)},
$$

where $L$ is the line bundle associated to the $\operatorname{spin}^{c}$ structure, where $N F$ is the normal bundle of $F$ in $M$ and has fiber dimension $2 m$, and where $\tilde{c}_{1}, \hat{A}$ and Euler are, respectively, the equivariant Chern class, A-roof class, and Euler class. The orientations are chosen so that the orientation of $F$ followed by that of $N F$ gives the orientation of $M$.

Before proceeding with the proof, let us say a word about the derivation of these formulas. Formula (7.1), with expression (7.2), follows from Theorem (3.9) in [ASIII] by a derivation similar to that of formula (5.4) in [ASIII]. The difference is that Atiyah and Singer work out the case of the Dirac operator associated to a spin structure, whereas we need the Dirac operator associated to a $\operatorname{spin}^{c}$ structure. We now explain, briefly, the necessary adjustments:

The weight lattice of the maximal torus in the group $\operatorname{spin}^{c}(2 n)=\operatorname{spin}(2 n) \times_{\mathbb{Z}_{2}}$ $U(1)$ is generated by the elements $x_{1}, \ldots, x_{n}, y$, and $\frac{1}{2}\left(x_{1}+\ldots+x_{n}+y\right)$, where $x_{i}$ and $y$ generate the weight lattices of the maximal tori in $S O(2 n)=\operatorname{spin}(2 n) / \mathbb{Z}_{2}$ and in $U(1) / \mathbb{Z}_{2}$. The $\operatorname{spin}^{c}$-Dirac operator is constructed out of the spinor representations $\Delta_{\mathbb{C}}^{+}:=\Delta^{+} \otimes \mathbb{C}$ and $\Delta_{\mathbb{C}}^{-}:=\Delta^{-} \otimes \mathbb{C}$ of the group spin ${ }^{c}$. Temporarily adopting the conventions of Atiyah and Singer, the weights occurring in these representations are $\frac{1}{2}\left( \pm x_{1} \ldots \pm x_{n}+y\right)$ with an even number of minus signs for weights in $\Delta_{\mathbb{C}}^{+}$and an odd number of minus signs for weights in $\Delta_{\mathbb{C}}^{-}$. This gives $\operatorname{ch}\left(\Delta_{\mathbb{C}}^{+}\right)-\operatorname{ch}\left(\Delta_{\mathbb{C}}^{-}\right)=e^{y / 2} \prod_{i=1}^{n}\left(e^{x_{i} / 2}-e^{-x_{i} / 2}\right)$ instead of formula (5.1) in [ASIII]. The formula at the top of [ASIII, p. 571] then gets multiplied by $e^{y / 2}$, giving index $D^{+}=(-1)^{n} \exp \left(\frac{1}{2} c_{1}(L)\right) \hat{A}(M)$. Finally, since our operator agrees with theirs if $n=\frac{1}{2} \operatorname{dim} M$ is even and disagrees if $n$ is odd (see Remark 2.3), the factor $(-1)^{n}$ disappears.

We also note that in formula (5.4) of [ASIII] the sign depends "in a subtle global manner on the particular component" [ASIII, p. 572]. This difficulty arises from the possible ambiguity in lifting a $G$-action from the manifold to its spin bundle. Since our group $G$ is connected, no such ambiguity arises.

We will now review the definitions of the characteristic classes that occur in expression (7.2).

First, if $L$ is a complex line bundle with ordinary Chern class $[\omega]$, and if the circle group acts on the fibers of $L$ with a weight $\alpha$, the equivariant Chern class of $L$ is $\omega+\alpha u$, where $u$ is a formal variable. See Appendix A.

The Lie algebra of the maximal torus of $\mathrm{SO}(2 n)$ is $\mathbb{R}^{n}$. The Weyl group acts by permuting the $x_{i}$ 's and by replacing $x_{i}, x_{j}$ by $-x_{i},-x_{j}$ for pairs of indices $i, j$. Any power series on $\mathbb{R}^{n}$ that is invariant under these operations determines a characteristic class on $\mathrm{SO}(2 n)$-principal bundles. In particular, the $\hat{A}$-class is 
determined from the power series expansion of the expression

$$
\hat{A}=\prod_{i=1}^{n} \frac{x_{i}}{e^{x_{i} / 2}-e^{-x_{i} / 2}},
$$

the Euler class is determined by the polynomial

$$
\text { Euler }=\prod_{i=1}^{n} x_{i}
$$

and their quotient is determined by the power series expansion of the expression

$$
\prod \frac{1}{e^{x_{i} / 2}-e^{-x_{i} / 2}}
$$

In (7.2), $\hat{A}(F)$ means the $\hat{A}$-class associated to the frame bundle $\mathcal{F}(T F)$, on which the circle action is trivial. For the normal bundle, $N F$, we need to work with the equivariant analogues of the above characteristic classes.

Since the circle acts on the fibers of $N F$ while fixing only the zero section, $N F$ is isomorphic (as an equivariant oriented real vector bundle) to a complex vector bundle. By the (equivariant) splitting principle we can pretend that $N F$ splits into a direct sum of complex line bundles, $N F=\bigoplus_{i} L_{i}$. The Chern-Weil recipe then dictates that in (7.5) we replace each $x_{i}$ by the equivariant Chern class, $\omega_{i}+\alpha_{i} u$, of $L_{i}$, where the $\omega_{i}$ and $\alpha_{i}$ are the ordinary Chern class and the circle weight on $L_{i}$. This yields

$$
\frac{\hat{A}(N F)}{\operatorname{Euler}(N F)}(u)=\prod_{i} \frac{1}{e^{\left(\omega_{i}+\alpha_{i} u\right) / 2}-e^{-\left(\omega_{i}+\alpha_{i} u\right) / 2}} .
$$

The sum $\sum_{F} \operatorname{AS}_{F}(u)$ is a polynomial in $z=\exp (u)$ and $z^{-1}=\exp (-u)$, because it is the character of the virtual representation $Q(M, P, p)$. The (virtual) dimension of the fixed space, $\operatorname{dim} Q(M, P, p)^{S^{1}}$, is equal to the coefficient of 1 in this polynomial.

The individual contribution, $\operatorname{AS}_{F}(u)$, while not a polynomial in $z$ and $z^{-1}$, does extend to a meromorphic function of $z \in \mathbb{C} \cup\{\infty\}$, and can be expanded into either a formal power series in $z^{-1}$ plus a polynomial in $z$, or a formal power series in $z$ plus a polynomial in $z^{-1}$. In either case, the dimension of the space of fixed vectors in $Q(M, P, p)$ is given by summing the coefficients of 1 over all $F$ 's.

We now expand (7.2) into a formal power series in $z^{-1}$. If $\alpha_{i}>0$, the $i$ th factor in (7.6) expands as

$$
e^{-\left(\omega_{i}+\alpha_{i} u\right) / 2}\left(1+c z^{-1}+c^{\prime} z^{-2}+\ldots\right) .
$$

(Here, and throughout the rest of this paper, $c, c^{\prime}, \ldots$ will serve as place holders for arbitrary cohomology classes; a priori, the $c$ 's in this formula bear no relation to any $c$ 's appearing later.) If $\alpha_{i}<0$, the $i$ th factor in (7.6) expands as

$$
-e^{\left(\omega_{i}+\alpha_{i} u\right) / 2}\left(1+c z^{-1}+c^{\prime} z^{-2}+\ldots\right) .
$$

Plugging into (7.2), we get

$$
A S_{F}(u)=(-1)^{m} z^{\frac{1}{2}\left(\alpha-\sum\left|\alpha_{i}\right|\right)} \int_{F} c+c^{\prime} z^{-1}+c^{\prime \prime} z^{-2}+\ldots .
$$


A similar computation, when we expand in powers of $z$, gives the following:

$$
A S_{F}(u)=(-1)^{m} z^{\frac{1}{2}\left(\alpha+\sum\left|\alpha_{i}\right|\right)} \int_{F} c+c^{\prime} z+c^{\prime \prime} z^{2}+\ldots
$$

The lemma below follows immediately.

Lemma 7.9. Let $S^{1}$ act on a smooth manifold $M$. Let $(P, p)$ be a an equivariant spin $^{c}$ structure on $M$.

1. When we expand the Atiyah-Segal-Singer index formula (7.1), (7.2) for the quantization $Q(M, P, p)$ as a formal power series in $z$, the fixed point component $F$ does not contribute to $\operatorname{dim} Q(M, P, p)^{S^{1}}$ if

$$
\text { fiber weight at } F>-\sum \mid \text { tangent weights at } F \mid \text {. }
$$

2. When we expand the Atiyah-Segal-Singer index formula for $Q(M, P, p)$ as a formal power series in $z^{-1}$, the fixed point component $F$ does not contribute to $\operatorname{dim} Q(M, P, p)^{S^{1}}$ if

$$
\text { fiber weight at } F<\sum \mid \text { tangent weights at } F \mid \text {. }
$$

We are now ready to prove our main theorem. Let $S^{1}$ act on a manifold $M$, and let $(P, p)$ be an equivariant $\operatorname{spin}^{c}$ structure. Let $Z$ be a reducible hypersurface that splits $M$ into $M_{+}$and $M_{-}$. Assume that conditions (4.8) are satisfied. Equivalently, assume that

$$
\begin{aligned}
& F \subset M_{+} \Rightarrow \text { fiber weight at } F>-\sum \mid \text { tangent weights at } F \mid, \\
& F \subset M_{-} \Rightarrow \text { fiber weight at } F<\sum \mid \text { tangent weights at } F \mid .
\end{aligned}
$$

We begin by considering the quantization of $M$. By Lemma 7.9, fixed points in $M_{-}$contribute nothing to $Q(M, P, p)^{S^{1}}$ when we take the expansion in $z^{-1}$; that is,

$$
\operatorname{dim} Q(M, P, p)^{S^{1}}=\sum_{F \subset M_{+}} \quad \begin{aligned}
& \text { coefficient of } 1 \text { in the expansion of } A S_{F}(u) \\
& \text { as a formal power series in } z^{-1} .
\end{aligned}
$$

Now consider the cut space, $M_{\text {cut }}$. By Proposition 6.1, part 1, the set of fixed points in $M_{\text {cut }}$ is the union

$$
M_{\text {cut }}^{S^{1}}=i_{+}\left(M_{+}^{S^{1}}\right) \sqcup i_{\text {red }}\left(M_{\text {red }}\right),
$$

where $M_{+}^{S^{1}}$ is the set of fixed points in $M_{+}$. Let us denote by $F^{\prime}$ and $X^{\prime}$, respectively, the images in $M_{\text {cut }}$ of connected components $F$ of $M_{+}^{S^{1}}$ and $X$ of $M_{\text {red. Then }}$

$$
\begin{aligned}
& \operatorname{dim} Q\left(M_{\text {cut }}, P_{\text {cut }}, p_{\text {cut }}\right)^{S^{1}} \\
& =\sum_{F \subset M_{+}} \begin{array}{l}
\text { coefficient of } 1 \text { in the expansion of } A S_{F^{\prime}}(u) \\
\text { as a formal power series in } z^{-1}
\end{array} \\
& \quad+\sum_{X \subset M_{\text {red }}} \quad \begin{array}{l}
\text { coefficient of } 1 \text { in the expansion of } A S_{X^{\prime}}(u) \\
\text { as a formal power series in } z^{-1} .
\end{array}
\end{aligned}
$$

Let $F$ be a connected component of $M_{+}^{S^{1}}$, and let $F^{\prime}:=i_{+}(F)$ be its image in $M_{\text {cut }}$. By Proposition 6.1, parts 1, 3(a), and 4(a), and by (7.2), the contribution of 
$F$ to the quantization of $M$ and the contribution of $F^{\prime}=i_{+}(F)$ to the quantization of $M_{\text {cut }}$ are identical:

$$
A S_{F^{\prime}}(u)=A S_{F}(u) .
$$

Let $X$ be a connected component of $M_{\text {red }}$, let $X^{\prime}:=i_{\text {red }}(X)$ be its image in $M_{\text {cut }}$, and let $N X^{\prime}$ be the normal bundle of $X^{\prime}$ in $M_{\text {cut }}$. Let $N:=Z \times_{S^{1}} \mathbb{C}$ be the line bundle over $X$ as in (6.2). The contribution (7.2) of the fixed component $X^{\prime}$ to the quantization of $M_{\text {cut }}$ is

$$
\begin{aligned}
A S_{X^{\prime}}(u) & =-\int_{X^{\prime}} \exp \left(\frac{1}{2} \tilde{c}_{1}\left(\left.L_{\text {cut }}\right|_{X^{\prime}}\right)\right) \hat{A}\left(X^{\prime}\right) \frac{\hat{A}\left(N X^{\prime}\right)}{\operatorname{Euler}\left(N X^{\prime}\right)} \\
& =-\int_{X} \exp \left(\frac{1}{2} \tilde{c}_{1}\left(L_{\text {red }} \otimes N\right)\right) \hat{A}(X) \frac{\hat{A}(N)}{\operatorname{Euler}(N)} \\
& =-\int_{X} \exp \left(\frac{1}{2}\left(c_{1}\left(L_{\text {red }}\right)\right)\right) \hat{A}(X) \frac{\exp \left(\frac{1}{2} \tilde{c}_{1}(N)\right) \hat{A}(N)}{\operatorname{Euler}(N)},
\end{aligned}
$$

where the middle equality follows from Proposition 6.1, parts 2, 3(b), and 4(b).

Since $N$ is a complex line bundle with fiber weight 1 , and by (7.6), we have

$$
\exp \left(\frac{1}{2} \tilde{c}_{1}(N)\right)=\exp \left(\frac{1}{2}(\omega+u)\right)
$$

and

$$
\frac{\hat{A}(N)}{\operatorname{Euler}(N)}=\frac{1}{\exp \left(\frac{1}{2}(\omega+u)\right)-\exp \left(-\frac{1}{2}(\omega+u)\right)}
$$

where $[\omega]$ is the first Chern class of $N$. Plugging this into (7.14) and expanding in powers of $z^{-1}=\exp (-u)$, we get

$$
A S_{X^{\prime}}(u)=-\int_{X} \exp \left(\frac{1}{2}\left(c_{1}\left(L_{\mathrm{red}}\right)\right)\right) \hat{A}(X)\left(1+c z^{-1}+c^{\prime} z^{-2}+\ldots\right) .
$$

By applying the (non-equivariant) Atiyah-Singer formula to $M_{\text {red }}$, we see that

$$
\begin{aligned}
& \sum_{X \subset M_{\mathrm{red}}} \begin{array}{l}
\text { coefficient of } 1 \text { in the expansion of } A S_{X^{\prime}}(u) \\
\text { as a formal power series in } z^{-1}
\end{array} \\
& =-\operatorname{dim} Q\left(M_{\text {red }}, P_{\text {red }}, p_{\text {red }}\right) \text {. }
\end{aligned}
$$

Comparing the expressions (7.11), (7.12), (7.13) and (7.15), we get

$$
\operatorname{dim} Q\left(M_{\text {cut }}, P_{\text {cut }}, p_{\text {cut }}\right)^{S^{1}}=\operatorname{dim} Q(M, P, p)^{S^{1}}-\operatorname{dim} Q\left(M_{\text {red }}, P_{\text {red }}, p_{\text {red }}\right) .
$$

Now consider the expansions in $z$. For every fixed component of the form $F^{\prime}=$ $i_{+}(F)$, for $F \subset M_{+}$, the first condition of (7.10) together with part 1 of Lemma 7.9 implies that this fixed point set does not contribute. Similarly, consider a fixed component of the form $X^{\prime}=i_{\text {red }}(X)$, for $X \subset M_{\text {red }}$. Its fiber weight is $\alpha=1$, by Proposition 6.1, part 2. Hence, by part 1 of Lemma 7.9, $X^{\prime}$ does not contribute either. Therefore,

$$
\operatorname{dim} Q\left(M_{\text {cut }}, P_{\text {cut }}, p_{\text {cut }}\right)^{S^{1}}=0 .
$$

Since (7.16) and (7.17) must be equal, we have

$$
\operatorname{dim} Q(M, P, p)^{S^{1}}=\operatorname{dim} Q\left(M_{\text {red }}, P_{\text {red }}, p_{\text {red }}\right),
$$

i.e., $\operatorname{spin}^{c}$ quantization commutes with reduction. 


\section{Appendix A. Remark on CONVEntions}

Let $\pi: P \rightarrow M$ be a principal $S^{1}$ bundle. A connection form on $P$ is an invariant one-form, $\Theta \in \Omega^{1}\left(P, \operatorname{Lie}\left(S^{1}\right)\right)$, that restricts to the canonical form on each fiber. Its curvature is the two-form $\Omega \in \Omega^{2}\left(M, \operatorname{Lie}\left(S^{1}\right)\right)$ such that $\pi^{*}(\Omega)=d \Theta$.

If $S^{1}$ acts on $P$, define a map $\Psi: M \rightarrow\left(\operatorname{Lie}\left(S^{1}\right)\right)^{*} \otimes \operatorname{Lie}\left(S^{1}\right)$ by $\pi^{*} \Psi(\xi)=\Theta\left(\xi_{P}\right)$, where $\xi_{P}$ is the vector field on $P$ corresponding to $\xi \in \operatorname{Lie}\left(S^{1}\right)$ under the action. Then $\Psi$ is a moment map for $\Omega$; that is, $d \Psi(\xi)=-\iota\left(\xi_{M}\right) \Omega$ for all $\xi \in \operatorname{Lie}\left(S^{1}\right)$, where $\xi_{M}$ is the vector field on $M$ that generates the action of $\exp (t \xi), t \in \mathbb{R}$.

Over each component $F$ of the fixed point set, this action gives a representation $\rho_{F}: S^{1} \rightarrow S^{1}$. The value of $\Psi$ at $F$ is precisely the weight of the representation $\rho_{F}$; no choices are necessary. Under the natural identification of $\operatorname{Lie}\left(S^{1}\right)^{*} \otimes \operatorname{Lie}\left(S^{1}\right)$ with $\mathbb{R}$, the weights are identified with the integers, $\mathbb{Z} \subset \mathbb{R}$, and $\Psi$ is identified with a real valued function $\Phi: M \rightarrow \mathbb{R}$.

In this paper, we always identify $\operatorname{Lie}\left(S^{1}\right)$ with $\mathbb{R}$ by the isomorphism that takes the positive primitive lattice element, $\eta \in \operatorname{Lie}\left(S^{1}\right)$, to -1 . The curvature, $\Omega$, is then identified with a real-valued two-form, $\omega$, which represents the first Chern class of $P$. The criterion for a moment map then becomes $d \Phi=\iota\left(\eta_{M}\right) \omega$.

\section{REFERENCES}

[AB1] M. Atiyah and R. Bott, A Lefschetz fixed point formula for elliptic complexes. II, Bull. Amer. Math. Soc. 87 (1968), 415-491. MR 38:731

[AB2] M. Atiyah and R. Bott, The moment map and equivariant cohomology, Topology 23 (1984), 1-28. MR 85e:58041

[ASII] M. Atiyah and G. Segal, The index of elliptic operators. II, Ann. of Math. 87 (1968), 531-545. MR 38:5246

[ASIII] M. F. Atiyah and I. M. Singer, The index of elliptic operators. III, Ann. of Math. 87 (1968), 546-604. MR 38:5245

[BGV] N. Berline, E. Getzler and M. Vergne, Heat Kernels and Dirac Operators, Springer, New York, 1992. MR 94e:58130

[BV] N. Berline and M. Vergne, Classes caractéristiques équivariantes, formule de localisation en cohomologie équivariante, C. R. Acad. Sci. Paris 295 (1982), 539-541. MR 83m:58002

[BS] A. Borel, J. P. Serre, Groupes de Lie et puissances réduites de Steenrod, Amer. J. Math. 75 (1953), 409-448. MR 15:338b

[BU] D. Borthwick and A. Uribe, Almost complex structures and geometric quantization, Math. Res. Lett. 3 (1996), no. 6, 845-861. MR 98e:58084

[B1] R. Bott, Homogeneous vector bundles, Ann. of Math. (2) 66 (1957), 203-248. MR 19:681d

[B2] R. Bott, The index theorem for homogeneous differential operators, Differential and Combinatorial Topology - a symposium in honor of Marston Morse (1965), 167-186. MR 31:624b

[BT] R. Bott and L. Tu, Differential Forms in Algebraic Topology, Springer, New York, 1982. MR 83i: 57016

[CGW] A. Cannas da Silva, V. Guillemin and C. Woodward, On the unfolding of folding symplectic structures, preprint, 1998.

[D] J. J. Duistermaat, The Heat Kernel Lefschetz Fixed Point Formula for the Spin-c Dirac Operator, Birkhäuser, Boston, 1996. MR 97d:58181

[DGMW] J. J. Duistermaat, V. Guillemin, E. Meinrenken and S. Wu, Symplectic reduction and Riemann-Roch for circle actions, Math. Res. Letters 2 (1995), 259-266.

[GGK] V. Ginzburg, V. Guillemin and Y. Karshon, Cobordism theory and localization formulas for Hamiltonian group actions, Int. Math. Res. Notices 5 (1996), 221-234. MR 97d:57046

[GNH] M. Gotay, J. Nester, and G. Hinds, Presymplectic manifolds and the Dirac-Bergmann theory of constraints, J. Math. Phys. 19 (11), Nov. 1978. MR 80e:58025 
[GK1] M. Grossberg and Y. Karshon, Bott towers, complete integrability and the extended character of representations, Duke Math. J. 76 no. 1, 23-58 (1994). MR 96i:22030

[GK2] M. Grossberg and Y. Karshon, Equivariant index and the moment map for completely integrable torus actions, Adv. Math. 133 (1998), 185-223. CMP 98:08

[G] V. Guillemin, Reduced phase-spaces and Riemann-Roch, In Lie groups and geometry in honor of B. Kostant (R. Brylinski et al., eds.), Progress in Mathematics 123, Birkhäuser, Boston (1995), 305-334. MR 96m:58095

[GS] V. Guillemin and S. Sternberg, Geometric quantization and multiplicities of group representations, Invent. Math. 67 (1982), 515-538. MR 83m:58040

[JK] L. Jeffrey and F. Kirwan, On localization and Riemann-Roch numbers for symplectic quotients, Quart. J. Math. Oxford Ser. (2) 47 (1996), no. 186, 165-185. MR 97i:58064

[KT] Y. Karshon and S. Tolman, The moment map and line bundles over pre-symplectic toric manifolds J. Differential Geometry 38 (1993), 464-484. MR 94j:58065

[K] M. Karoubi, K-Theory, An Introduction, Springer, New York, 1978. MR 58:7605

[Ko] B. Kostant, Quantization and unitary representations, in Modern Analysis and Applications, Springer Lecture Notes in Mathematics, No. 170, pp. 87-107, New York, Springer-Verlag, 1970. MR 45:3638

[LM] H. Lawson and M. Michelson, Spin Geometry, Princeton, 1989.

[L] E. Lerman, Symplectic cuts, Math. Res. Letters 2 (1995), 247-258. MR 96f:58062

[MW] J. Marsden and A. Weinstein, Reduction of symplectic manifolds with symmetry, Rep. Math. Phys. 5 (1974), 121-130. MR 53:6633

[M1] E. Meinrenken, On Riemann-Roch formulas for multiplicities, J. Amer. Math. Soc. 9 (1996), no. 2, 373-389. MR 96f:58070

[M2] E. Meinrenken, Symplectic surgery and the spin ${ }^{c}$-Dirac operator, Adv. Math. 134 (1998), 240-277. CMP 98:11

[MS] E. Meinrenken and R. Sjamaar, Singular reduction and quantization, Topology 38 (1999), 699-762.

[P] R. Parthasarathy, Dirac operator and the discrete series, Annals of Math. 96 (1972), 1-30. MR 47:6945

[S] R. Sjamaar, Symplectic reduction and Riemann-Roch formulas for multiplicities, Bull. Amer. Math. Soc. 33 (1996), 327-338. MR 96j:58073

[T] S. Tolman, in preparation.

[V1] M. Vergne, Geometric quantization and equivariant cohomology, First European Congress in Mathematics, vol. 1, Progress in Mathematics 119, Birkhäuser, Boston (1994), 249-295. MR 96j:58074

[V2] M. Vergne, Multiplicity formula for geometric quantization. I, II, Duke Math. Journal, 82 (1996), No. 1, 143-179 and 181-194. MR 98e:58087

Department of Mathematics, University of California, Berkeley, California 94720

E-mail address: acannas@math.berkeley.edu

Institute of Mathematics, The Hebrew University, Jerusalem 91904, IsRael

E-mail address: karshon@math.huji.ac.il

Department of Mathematics, Princeton University, Princton, New Jersey 08544-1000

E-mail address: tolman@math.princeton.edu 